\title{
Minmax Tree Cover in the Euclidean Space
}

\author{
Seigo Karakawa $\left.\right|^{1}$ Ehab Morsy $\left.\right|^{1}$ Hiroshi Nagamochi $\left.\right|^{1}$ \\ ${ }^{1}$ Department of Applied Mathematics and Physics \\ Graduate School of Informatics, \\ Kyoto University, Yoshida Honmachi, Sakyo, \\ Kyoto 606-8501, Japan
}

\begin{abstract}
Let $G=(V, E)$ be an edge-weighted graph, and let $w(H)$ denote the sum of the weights of the edges in a subgraph $H$ of $G$. Given a positive integer $k$, the balanced tree partitioning problem requires to cover all vertices in $V$ by a set $\mathcal{T}$ of $k$ trees of the graph so that the ratio $\alpha$ of $\max _{T \in \mathcal{T}} w(T)$ to $w\left(T^{*}\right) / k$ is minimized, where $T^{*}$ denotes a minimum spanning tree of $G$. The problem has been used as a core analysis in designing approximation algorithms for several types of graph partitioning problems over metric spaces, and the performance guarantees depend on the ratio $\alpha$ of the corresponding balanced tree partitioning problems. It is known that the best possible value of $\alpha$ is 2 for the general metric space. In this paper, we study the problem in the $d$-dimensional Euclidean space $\mathbb{R}^{d}$, and break the bound 2 on $\alpha$, showing that $\alpha<2 \sqrt{3}-3 / 2 \fallingdotseq 1.964$ for $d \geq 3$ and $\alpha<(13+\sqrt{109}) / 12 \fallingdotseq 1.953$ for $d=2$. These new results enable us to directly improve the performance guarantees of several existing approximation algorithms for graph partitioning problems if the metric space is an Euclidean space.
\end{abstract}

\begin{tabular}{|cccc|}
\hline $\begin{array}{c}\text { Submitted: } \\
\text { April 2009 }\end{array}$ & Reviewed: & Revised: & Accepted: \\
& September 2009 & July 2010 & October 2010 \\
& Final: & Published: \\
October 2010 & July 2011 \\
Article type: & Communicated by: \\
Regular paper & S. Das and R. Uehara \\
\hline
\end{tabular}

A preliminary version of the paper appeared in Proceedings of the Third Annual Workshop on Algorithms and Computation (WALCOM), Feb. 18-20, 2009, Kolkata, India, LNCS 5431 (2009) 202-213 .

This research was partially supported by the Scientific Grant-in-Aid from Ministry of Education, Culture, Sports, Science and Technology of Japan.

E-mail addresses: seigo@amp.i.kyoto-u.ac.jp (Seigo Karakawa) ehab@amp.i.kyoto-u.ac.jp (Ehab Morsy) nag@amp.i.kyoto-u.ac.jp (Hiroshi Nagamochi) 
346 S. Karakawa, E. Morsy, and H. Nagamochi Minmax Tree Cover in the Euclidean Space

\section{Introduction}

Let $G=(V, E)$ be a simple undirected graph with vertex set $V$ and edge set $E$ such that edges are weighted by nonnegative reals, and let $p$ be a specified positive integer. Then the minmax subtree cover problem requires to find a set of $p$ trees covering all the vertices such that the maximum weight of a tree in the set is minimized. This problem arises in various types of practical applications, such as multi-vehicle scheduling problem [6, 8, 9, 10, task sequencing problem [4, and political districting [3, 7. The minmax subtree cover problem on graphs can be described formally as follows, where $\mathbb{R}^{+}$denotes the set of nonnegative reals.

Minmax Subtree Cover Problem (MSC):

Input: An undirected graph $G=(V, E)$, an edge weight function $w: E \rightarrow \mathbb{R}^{+}$, and a positive integer $p$.

Feasible solution: A partition $\mathcal{S}=\left\{S_{1}, S_{2}, \ldots, S_{p}\right\}$ of $V$ and a set $\mathcal{T}=$ $\left\{T_{1}, T_{2}, \ldots, T_{p}\right\}$ of $p$ trees of $G$ such that $S_{i} \subseteq V\left(T_{i}\right), i=1,2, \ldots, p$.

Goal: Minimize $\max _{1 \leq i \leq p} w\left(T_{i}\right)$, where $w\left(T_{i}\right)=\sum_{e \in E\left(T_{i}\right)} w(e)$.

A tree cover $\mathcal{T}$ of a graph $G=(V, E)$ is defined by a set of trees of $G$ such that the union of vertices of the trees in $\mathcal{T}$ contains $V$. That is, the MSC requires to find a tree cover with $p$ trees that minimizes the maximum weight of a tree in the tree cover, where the weight of a tree is the sum of the weights of all edges in the tree. Trees in a tree cover are not necessarily vertex-disjoint or edge-disjoint.

The MSC is known to be NP-hard even if $p=2$ and $G$ is a tree, or if $G$ can be embedded in the Euclidean space [1, 5, and thus several approximation algorithms for the problem have been proposed in the literatures. Andersson et al. 11] provided a $(2+\varepsilon)$-approximation algorithm for the MSC when $G$ can be embedded in the Euclidean space, where $\varepsilon$ is a sufficiently small positive real number, and Nagamochi and Kawada 13 presented a $(4-4 /(p+1))$ approximation algorithm for the problem when the given graph is a cactus. Even et al. 6] presented a 4-approximation algorithm for the MSC with an arbitrary graph $G$. The MSC on a tree-like structure graphs has also been studied extensively. Averbakh and Berman 2 presented a $(2-2 /(p+1))$-approximation algorithm with time complexity $O\left(p^{p-1} n^{p-1}\right)$, and afterward Nagamochi and Okada 14] gave a polynomial time approximation algorithm with the same approximation factor which runs in $O\left(p^{2} n\right)$ time, where $n$ is the number of vertices in the tree. In an extension of the problem, a set of vertices to be covered is given as a subset $S \subseteq V$ of vertices and nonnegative weights to handle vertices in $S$ are also introduced in the tree weight. For this problem, Nagamochi and Okada [15] proposed a $(2-2 /(p+1))$-approximation algorithm that runs in $O((p-1) ! n)$.

For a rooted version of the MSC, a graph $G=(V, E)$ with a set $R$ of prescribed vertices is given and a tree cover is required to consist of trees each of which is rooted at a vertex in $R$. In [6], a 4-approximation algorithm is proposed for an arbitrary graph $G$ under an additional condition that all trees $\mathcal{T}$ are 
required to be rooted at distinct vertices in $R$. Nagamochi 12 proposed a $(3-$ $2 /(p+1))$-approximation algorithm to the problem with $|R|=1$. For the rooted version of the MSC on a tree, Averbakh and Berman [1] presented a linear time $4 / 3$-approximation algorithm for the problem with $p=2$, and Nagamochi and Okada [14, 16] gave an $O\left(n \log \log _{1+\varepsilon / 2} 3\right)$ time $(2+\varepsilon)$-approximation algorithms for arbitrary $p$, where $\varepsilon>0$ is a prescribed constant.

Some of the above approximation algorithms for the MSC include a procedure for partitioning a minimum spanning tree $T^{*}$ of a given graph into $k$ trees of the graph as uniformly as possible, and their performance analysis relies on the fact that $w\left(T^{*}\right) / k$ is a lower bound on the maximum weight of a tree in any such partition. Thus the ratio $\alpha$ of the maximum weight of a tree to $w\left(T^{*}\right) / k$ appears as a factor of their performance guarantees. Motivated by the importance of the analysis on tree covers, we consider the balanced tree partitioning problem in this paper. For a given positive integer $k$, the problem requires to find a tree cover $\mathcal{T}=\left\{T_{1}, T_{2}, \ldots, T_{k}\right\}$ with $k$ trees of the graph that minimizes the ratio of $\max _{1 \leq i \leq k} w\left(T_{i}\right)$ to $w\left(T^{*}\right) / k$, where $T^{*}$ denotes a minimum spanning tree of $G$. Note that a tree $T_{i}$ in a tree cover is allowed to contain an edge not in $T^{*}$. In particular, we analyze an upper bound on the factor $\alpha$ such that

$$
\max _{1 \leq i \leq k} w\left(T_{i}\right) \leq \alpha \cdot w\left(T^{*}\right) / k .
$$

For any metric, it is known that $\alpha \leq 2$ holds [1, 2]. We here remark that $\alpha \leq 2$ is best possible for the general metric. Consider a metric graph $G=$ $(V, E)$ consisting of a star $S$ on $k+2$ vertices $V=\left\{s, v_{1}, \ldots, v_{k+1}\right\}$ such that $E(S)=\left\{\left(s, v_{i}\right) \mid i=1,2, \ldots, k+1\right\}$, where each edge in $S$ is weighted by 1 and each of the remaining edges in $G$ is weighted by 2. Clearly, $S$ is the minimum spanning tree of $G$ with weight $w(S)=k+1$. On the other hand, the maximum weight of any $k$ trees of $G$ is at least 2 , since one of the trees must contain two leaves of $S$. Hence $\alpha$ is at least $2 /(1+1 / k)$, which is nearly 2 when $k$ is sufficiently large.

In this paper, we prove that there are better upper bounds on $\alpha$ in the Euclidean space. The following two results are the main contribution of the paper.

Theorem 1 For a set $V$ of $n$ points in the Euclidean space $\mathbb{R}^{2}$ and a positive integer $k$, there exists a tree cover $T_{1}, T_{2}, \ldots, T_{k}$ of $V$ such that

$$
\frac{\max _{1 \leq i \leq k} w\left(T_{i}\right)}{w\left(T^{*}\right) / k} \leq(13+\sqrt{109}) / 12 \fallingdotseq 1.953,
$$

where $T^{*}$ is a minimum weight tree spanning $V$. Furthermore, such a tree cover can be obtained in polynomial time.

Theorem 2 For a set $V$ of $n$ points in the Euclidean space $\mathbb{R}^{d}(d \geq 3)$ and a positive integer $k$, there exists a tree cover $T_{1}, T_{2}, \ldots, T_{k}$ of $V$ such that

$$
\frac{\max _{1 \leq i \leq k} w\left(T_{i}\right)}{w\left(T^{*}\right) / k} \leq 2 \sqrt{3}-3 / 2 \fallingdotseq 1.964
$$


348 S. Karakawa, E. Morsy, and H. Nagamochi Minmax Tree Cover in the Euclidean Space

where $T^{*}$ is a minimum weight tree spanning $V$. Furthermore, such a tree cover can be obtained in polynomial time.

These new results enable us to directly improve the performance guarantees of several existing approximation algorithms for graph partitioning problems if the metric space is a Euclidean space $\mathbb{R}^{d}$. For example, the performance guarantee on the MSC due to Andersson et al. [11 is given as $\alpha+\varepsilon$, which is at most $1.965+\varepsilon$ for $d \geq 3$ and $1.954+\varepsilon$ for $d=2$. Also the performance guarantees on the rooted and unrooted versions of the MSC due to Even et al. [6] are $2+\alpha$ and $2 \alpha$, respectively. Thus, our results imply that the unrooted versions of the MSC is 3.929-approximable in $\mathbb{R}^{d}$ with $d \geq 3$ and 3.907-approximable in $\mathbb{R}^{2}$.

The paper is organized as follows. Section 2 introduces terminologies and definitions on graphs. Section 3 gives a framework of an approximation algorithm to the balanced tree partitioning problem, from which Theorems 1 and 2 follow directly. Sections 4 , 5 and 6 give detailed proofs of some tree cover results based on which we build our algorithm in Section 3 . Section 7 makes some concluding remarks.

\section{Preliminaries}

Throughout this paper a graph stands for a simple undirected graph unless otherwise stated.

For a set $Z$, a set $\left\{Z_{1}, Z_{2}, \ldots, Z_{\ell}\right\}$ of pairwise disjoint non-empty subsets of $Z$ is called a partition of $Z$ if $\cup_{i=1}^{\ell} Z_{i}=Z$.

A singleton set $\{x\}$ may be denoted by $x$. Let $G$ be a graph. The vertex and edge sets of $G$ are denoted by $V(G)$ and $E(G)$, respectively. A graph $G$ with a vertex set $V$ and an edge set $E$ is denoted by $(V, E)$. Let $G_{1}$ and $G_{2}$ be subgraphs of $G$. We let $G_{1}+G_{2}$ denote the graph $\left(V\left(G_{1}\right) \cup V\left(G_{2}\right), E\left(G_{1}\right) \cup E\left(G_{2}\right)\right)$. For an edge $e=(u, v) \in E(G)$, we denote by $G_{1}+u$ (resp., $G_{1}+e$ ) the subgraph $G_{1}+(\{u\}, \emptyset)$ (resp., $\left.G_{1}+(\{u, v\},\{e\})\right)$ of $G$.

For a subset $X \subseteq V(G)$, let $G[X]$ denote the subgraph induced from $G$ by $X$. and $G-X$ denote the subgraph obtained from $G$ by removing the vertices in $X$ together with all edges incident to a vertex in $X$. The degree of $u$, i.e., the number of edges incident to vertex $u \in V(G)$, is denoted by $\operatorname{deg}(u)$.

For a graph $G$ and an edge weight $w: E(G) \rightarrow \mathbb{R}^{+}$, the weight $w\left(G^{\prime}\right)$ of a subgraph $G^{\prime}$ of $G$ is defined by $\sum_{e \in E\left(G^{\prime}\right)} w(e)$. For a set $\mathcal{S}$ of subgraphs of $G$, let $w(\mathcal{S})$ denote $\sum_{G^{\prime} \in \mathcal{S}} w\left(G^{\prime}\right)$. The weight $w(e)$ of edge $e=(u, v)$ may be denoted by $w(u, v)$. An edge-weighted graph $(G, w)$ is a metric if it satisfies the triangle inequality, i.e., $w(x, y)+w(y, z) \geq w(x, z)$ holds for all vertices $x, y, z \in V$. An edge-weighted graph $(G, w)$ in the Euclidean space $\mathbb{R}^{d}$ is a complete graph whose vertex set is defined by a set $V$ of points in the space, where the edge weight $w(u, v), u, v \in V$ is defined by the Euclidean distance between the two points $u$ and $v$. The line segment between points $u$ and $v$ in the Euclidean space $\mathbb{R}^{d}$ is denoted by $u v$ and its weight by $\overline{u v}$. 
Let $T$ be a rooted tree. The set of children of a vertex $v$ is denoted by $C h(v)$, and the set of descendants of a vertex $v$ is denoted by $D(v)$, where $D(v)$ includes $v$. Let $D(S)=\cup_{v \in S} D(v)$ for a subset $S \subseteq V(T)$. A non-root vertex of degree 1 is called a leaf in $T$. The subtree $T_{v}$ rooted at a vertex $v \in V(T)$ is the subtree of $T$ induced by the descendants of $v$. An edge $e=(u, v) \in E(T)$, where $v \in C h(u)$, is called the parent edge of $v$ and a child edge of $u$, and the subtree $T_{e}$ rooted at $e$ is defined by $T_{v}+e$. The parent edge of a vertex $v$ is denoted by $e(v)$. A branch of a vertex $u$ is defined as the subtree $T_{e}$ rooted at a child edge $e$ of $u$, and $\mathcal{B}(u)$ denotes the set of all branches of $u$. We may mean by a subset $\mathcal{S} \subseteq \mathcal{B}(u)$ the subtree that consists of all branches in $\mathcal{S}$ and denote by $V(\mathcal{S})$ and $E(\mathcal{S})$ the vertex and edge sets of the subtree $\mathcal{S}$, respectively, unless confusion arises.

For a real number $\beta>0$, we define a $\beta$-boundary vertex of $T$ as a non-root vertex $v$ such that

$$
w\left(T_{e(v)}\right) \geq \beta \text { for its parent edge } e(v),
$$

and no proper descendant of $v$ has this property, i.e.,

$$
w\left(T_{e^{\prime}}\right)<\beta \text { for every child edge } e^{\prime} \text { of } v \text { (if any). }
$$

For a subtree $T^{\prime}$ of $T$, let $V_{\beta}\left(T^{\prime}\right)$ denote the set of all $\beta$-boundary vertices in $T^{\prime}$, where the root $r^{\prime}$ of $T^{\prime}$ is chosen as the vertex closest to the root $r$ of $T$. Note that, for two distinct $\beta$-boundary vertices $u, v \in V_{\beta}\left(T^{\prime}\right)$, none of $u$ and $v$ is a descendent or an ancestor of the other in $T^{\prime}$. Also, $r^{\prime} \notin V_{\beta}\left(T^{\prime}\right)$ by definition. We observe the next property.

Lemma 1 Let $T_{e}$ be a branch of a vertex $u$ and $\beta$ be a positive real number.

(i) $V_{\beta}\left(T_{e}\right) \neq \emptyset$ if and only if $w\left(T_{e}\right) \geq \beta$.

(ii) If $V_{\beta}\left(T_{e}\right) \neq \emptyset$, then $V_{\gamma}\left(T_{e}\right) \neq \emptyset$ for any $\gamma \in(0, \beta]$.

Proof: (i) The only-if part is obvious. We show the if part. Choose a vertex $v \in V\left(T_{e}\right)-u$ closest to $u$ such that $v$ is a leaf or $w(S)<\beta$ holds for all $S \in \mathcal{B}(v)$. For the parent edge $e(v)$ of $v$, we have $w\left(T_{e(v)}\right) \geq \beta$. Then $v$ is a $\beta$-boundary vertex in $T_{e}$, and $V_{\beta}\left(T_{e}\right) \neq \emptyset$ holds.

(ii) For any real number $\gamma \in(0, \beta], w\left(T_{e}\right) \geq \beta \geq \gamma>0$ holds. Then $V_{\gamma}\left(T_{e}\right) \neq \emptyset$ holds by (i).

\section{Approximation Algorithm}

To prove Theorems 1 and 2 , we assume without loss of generality that $w\left(T^{*}\right)=k$ throughout the paper for a minimum spanning tree $T^{*}$. We design an approximation algorithm to the balanced tree partitioning problem to derive the upper bounds Theorems 1 and 2 . In this section, we first give a framework of the approximation algorithm. The algorithm computes a set of at most $k$ trees of 
350 S. Karakawa, E. Morsy, and H. Nagamochi Minmax Tree Cover in the Euclidean Space

$G$ that cover the vertex set of $T^{*}$ by repeatedly applying three main theorems on tree partition, where the proofs of such theorems will be given in the three subsequent sections.

We first introduce the following basic definitions.

For a specified real number $\alpha>3 / 2$ and a 1-boundary vertex $u \in V_{1}(T)$ in a rooted tree $T$, we define a canonical partition $\{\mathcal{L}(u), \mathcal{M}(u), \mathcal{H}(u)\}$ of $\mathcal{B}(u)$ by

$$
\begin{aligned}
\mathcal{L}(u) & =\{S \in \mathcal{B}(u) \quad \mid 0<w(S)<2-\alpha\}, \\
\mathcal{H}(u) & =\{S \in \mathcal{B}(u) \quad \mid \alpha-1<w(S)<1\}, \\
\mathcal{M}(u) & =\{S \in \mathcal{B}(u) \quad \mid 2-\alpha \leq w(S) \leq \alpha-1\} .
\end{aligned}
$$

Definition 3 Let $T$ be a tree in a graph $G$, and let $\alpha \in[1,2]$ be a real number. A family of $h$ subsets $S_{1}, S_{2}, \ldots, S_{h} \subseteq V(T)$ is admissible (or $h$-admissible) in $T$ if there are trees $T_{S_{1}}, T_{S_{2}}, \ldots, T_{S_{h}}$ of $G$ (which are not necessarily subtrees of T) such that

(i) For each $S_{i}, S_{i} \subseteq V\left(T_{S_{i}}\right)$ and $w\left(T_{S_{i}}\right) \leq \alpha$.

(ii) If $V(T)-\bigcup_{1 \leq i \leq h} S_{i} \neq \emptyset$, then $T^{\prime}=T-\bigcup_{1 \leq i \leq h} S_{i}$ remains connected and $w(T)-w\left(T^{\prime}\right) \geq h$ holds.

(iii) If $V(T)=\bigcup_{1 \leq i \leq h} S_{i}$, then $\lceil w(T)\rceil \geq h$ holds.

We call a set of such subtrees $T_{S_{i}}, i=1,2, \ldots, h$, an $h$-admissible forest.

To prove Theorems 1 and 2 where the weight of a minimum spanning tree $T^{*}$ of $G$ is assumed to be $k$, it suffices to show that a minimum spanning tree $T=T^{*}$ has a $k$-admissible family of subsets $S_{1}, S_{2}, \ldots, S_{k} \subseteq V(G)=V(T)$ for $\alpha=(13+\sqrt{109}) / 12$ and $\alpha=2 \sqrt{3}-3 / 2$, respectively, and such a family is polynomially computable.

Lemma 2 Let $T$ be a tree with $w(T)>0$ and $\alpha \in[1,2]$ be a specified real number.

(i) For a p-admissible family $\left\{S_{1}, S_{2}, \ldots, S_{p}\right\}$ in $T$ and a q-admissible family $\left\{S_{1}^{\prime}, S_{2}^{\prime}, \ldots, S_{q}^{\prime}\right\}$ in $T^{\prime}=T-\bigcup_{1 \leq i \leq p} S_{i}$, their union $\left\{S_{1}, S_{2}, \ldots, S_{p}\right\} \cup$ $\left\{S_{1}^{\prime}, S_{2}^{\prime}, \ldots, S_{q}^{\prime}\right\}$ is $(p+q)$-admissible in $T$.

(ii) For a non-root vertex $v \in V(T)$ with $w\left(T_{v}\right) \leq \alpha$ and $w\left(T_{e(v)}\right) \geq 1, D(v)$ is 1-admissible in $T$.

(iii) For a vertex $u \in V(T)$ and a subset $C_{1} \subseteq C h(u)$ with $1 \leq \sum_{v \in C_{1}} w\left(T_{e(v)}\right) \leq$ $\alpha, S_{1}=D\left(C_{1}\right)$ is 1-admissible in $T$.

(iv) If $w(T) \leq 3 \alpha / 2$, then there is an $h$-admissible family $\left\{S_{i} \mid i=1, \ldots, h\right\}$ $(h \leq 2)$ in $T$ such that $V(T)=\cup_{1 \leq i \leq h} S_{i}$. 
Proof: (i) From Definition $3\left(\right.$ i), there are trees $T_{S_{1}}, \ldots, T_{S_{p}}$ (resp., $T_{S_{1}^{\prime}}, \ldots, T_{S_{q}^{\prime}}$ ) of $G$ such that, for each $S_{i}$ (resp., $\left.S_{i}^{\prime}\right), w\left(T_{S_{i}}\right) \leq \alpha$ (resp., $w\left(T_{S_{i}^{\prime}}\right) \leq \alpha$ ). Also, Definition 3(ii) implies that $T^{\prime}-\bigcup_{1<i<q} S_{i}^{\prime}$ is connected and $w(T)-w\left(T^{\prime}\right) \geq p$ holds. Finally, if $V(T)=\left(\bigcup_{1<i<p} S_{i}\right) \cup\left(\bigcup_{1<i<q} S_{i}^{\prime}\right)$, then $\lceil w(T)\rceil=\lceil w(T)-$ $\left.w\left(T^{\prime}\right)+w\left(T^{\prime}\right)\right\rceil \geq\left\lceil p+w\left(T^{\prime}\right)\right\rceil \stackrel{=}{=}+\left\lceil w\left(T^{\prime}\right)\right\rceil \geq p+q$ holds (since $\left\lceil w\left(T^{\prime}\right)\right\rceil \geq q$ ). This proves (i).

Conditions (ii)-(iii) follow immediately from Definition 3 .

(iv) If $w(T) \leq \alpha$, then we see that $\left\{S_{1}=V(T)\right\}$ is admissible in $T$ and $T_{S_{1}}=T$ is a 1 -admissible tree.

Assume that $\alpha<w(T) \leq 3 \alpha / 2$. Regard $T$ as a tree rooted at a vertex $r$ with $\operatorname{deg}(r)=1$. For $\beta=w(T)-\alpha(>0), V_{\beta}(T) \neq \emptyset$ by Lemma 1(i). Choose a $\beta$-boundary vertex $u \in V_{\beta}(T)$ (note that $u \neq r$ ). We distinguish two cases, (1) $w\left(T_{u}\right)<\beta$ and $(2) w\left(T_{u}\right) \geq \beta$.

(1) $w\left(T_{u}\right)<\beta$. We show that $\left\{S_{1}=D(u), S_{2}=V(T)-S_{1}\right\}$ is admissible. Let $T_{S_{1}}:=T_{u}$ and $T_{S_{2}}:=T-V\left(T_{u}\right)$. Clearly

$$
w\left(T_{S_{1}}\right)=w\left(T_{u}\right)<\beta=w(T)-\alpha \leq 3 \alpha / 2-\alpha<\alpha .
$$

By the definition of a $\beta$-boundary vertex and $u \in V_{\beta}(T), w\left(T_{e(u)}\right) \geq \beta$ holds. Then we have

$$
w\left(T_{S_{2}}\right)=w(T)-w\left(T_{e(u)}\right) \leq w(T)-\beta=\alpha .
$$

Hence $\left\{T_{S_{1}}, T_{S_{2}}\right\}$ is a 2 -admissible forest, as required.

(2) $w\left(T_{u}\right) \geq \beta$. Choose a minimal subset $\mathcal{S} \subseteq \mathcal{B}(u)$ such that $w(\mathcal{S}) \geq \beta$ holds. We show that $\left\{S_{1}=V(\mathcal{S})-\{u\}, S_{2}=V(T)-S_{1}\right\}$ is admissible. Let $T_{S_{1}}:=\mathcal{S}$ and $T_{S_{2}}:=T-S_{1}$. By $u \in V_{\beta}(T)$, the weight of every branch of $u$ is less than $\beta$. Hence the minimality of $\mathcal{S}$ implies that

$$
\beta \leq w\left(T_{S_{1}}\right)=w(\mathcal{S})<\beta+\beta=2(w(T)-\alpha) \leq 2(3 \alpha / 2-\alpha)=\alpha .
$$

On the other hand, by $w(\mathcal{S}) \geq \beta$, we have

$$
w\left(T_{S_{2}}\right)=w(T)-w(\mathcal{S}) \leq w(T)-\beta=\alpha .
$$

Hence $\left\{T_{S_{1}}, T_{S_{2}}\right\}$ is a 2 -admissible forest, as required.

We consider whether a 1-boundary vertex $u$ in a tree satisfies the following condition.

Definition 4 For a real number $\alpha>3 / 2$, a 1-boundary vertex $u \in V_{1}(T)$ in a rooted tree $T$ is called inactive if it satisfies the following condition $A$, and is called active otherwise.

\section{Condition A:}

(i) $w(\mathcal{B}(u))>\alpha$, (ii) $|\mathcal{H}(u)| \geq 2$, (iii) $\mathcal{M}(u)=\emptyset$, and (iv) $w(\mathcal{L}(u))<2-\alpha$.

We use the following results, which will be verified in Sections 45 and 6 . respectively. 
352 S. Karakawa, E. Morsy, and H. Nagamochi Minmax Tree Cover in the Euclidean Space

Theorem 5 Let $T$ be a tree rooted at a vertex $r$ with $\operatorname{deg}(r)=1$, and let $\alpha \in[5 / 3,2]$ be a real number. Assume that $w(T)>3 \alpha / 2$ and there is an active 1-boundary vertex in $V_{1}(T)$. Then there is an admissible family $\mathcal{S}$ in $T$ such that the tree $T^{\prime}=T-\bigcup_{S \in \mathcal{S}} S$ has no active 1-boundary vertices in $V_{1}\left(T^{\prime}\right)$. Moreover, if $T^{\prime} \neq \emptyset, w\left(T^{\prime}\right)=0$, and $w(T[S \cup\{r\}])>\alpha$ for all $S \in \mathcal{S}$, then $\mathcal{S} \cup V\left(T^{\prime}\right)$ is an admissible family in $T$.

Theorem 6 Let $T$ be a tree in the Euclidean space $\mathbb{R}^{d}(d \geq 3)$, and let $\alpha=$ $2 \sqrt{3}-3 / 2$. Assume that $T$ is rooted at a vertex $r$ with $\operatorname{deg}(r)=1$. If $w(T)>$ $3 \alpha / 2$ and every 1-boundary vertex $u \in V_{1}(T)$ is inactive, then there is an admissible family $\mathcal{S}$ in $T$. Moreover, for $T^{\prime}=T-\bigcup_{S \in \mathcal{S}} S$, if $T^{\prime} \neq \emptyset, w\left(T^{\prime}\right)=0$, and $w(T[S \cup\{r\}])>\alpha$ for all $S \in \mathcal{S}$, then $\mathcal{S} \cup V\left(T^{\prime}\right)$ is an admissible family in $T$.

Theorem 7 Let $T$ be a tree in the Euclidean space $\mathbb{R}^{d}(d=2)$, and let $\alpha=$ $(13+\sqrt{109}) / 12$. Assume that $T$ is rooted at a vertex $r$ with $\operatorname{deg}(r)=1$. If $w(T)>3 \alpha / 2$ and every 1-boundary vertex $u \in V_{1}(T)$ is inactive, then there is an admissible family $\mathcal{S}$ in $T$. Moreover, for $T^{\prime}=T-\bigcup_{S \in \mathcal{S}} S$, if $T^{\prime} \neq \emptyset$, $w\left(T^{\prime}\right)=0$, and $w(T[S \cup\{r\}])>\alpha$ for all $S \in \mathcal{S}$, then $\mathcal{S} \cup V\left(T^{\prime}\right)$ is an admissible family in $T$.

Now we are ready to present the approximation algorithm. We are given a spanning tree $T$ of a graph $G$ in the Euclidean space and a positive integer $k$. The algorithm repeats the following procedure on the current tree $T$ as long as $w(T)>3 \alpha / 2$ holds. Depending on the properties of the current tree $T$, we first apply one of the above theorems to find an admissible family $\mathcal{S}$ in $T$ and then remove $\mathcal{S}$ from the current tree $T$. Finally, depending on the nature of the current tree $T$, we construct the desired admissible family in the given spanning tree. The algorithm is described as follows.

\section{Algorithm TreeCover}

Input: A spanning tree $T^{*}$ of a graph $G$ in the Euclidean space $\mathbb{R}^{d}(d \geq 2)$, where $w\left(T^{*}\right)=k$ for an integer $k \geq 1$.

Output: A $k$-admissible family $\mathcal{S}^{*}$ for $\alpha=(13+\sqrt{109}) / 12(d=2)$ and $\alpha=2 \sqrt{3}-3 / 2(d \geq 3)$.

1 Let $T:=T^{*}$, regarding $T$ as a tree rooted at a vertex $r$ with $\operatorname{deg}(r)=1$, and $\mathcal{S}^{*}:=\emptyset$;

2 while $w(T)>3 \alpha / 2$ do

3 if $T$ has an active 1-boundary vertex then

$4 \quad$ Find an admissible family $\mathcal{S}$ in $T$ by Theorem 5

$5 \quad$ Let $\mathcal{S}^{*}:=\mathcal{S}^{*} \cup \mathcal{S} ; T:=T-\bigcup_{S \in \mathcal{S}} S$

6 end; $/ *$ if $* /$

$/ *$ the current tree $T$ has no active 1-boundary vertex */

$7 \quad$ Find an admissible family $\mathcal{S}$ in $T$ by Theorem 6 (if $d \geq 3$ ) and Theorem 7 (if $d=2$ );

$8 \mathcal{S}^{*}:=\mathcal{S}^{*} \cup \mathcal{S} ; T:=T-\bigcup_{S \in \mathcal{S}} S$

9 end; /* while */ 


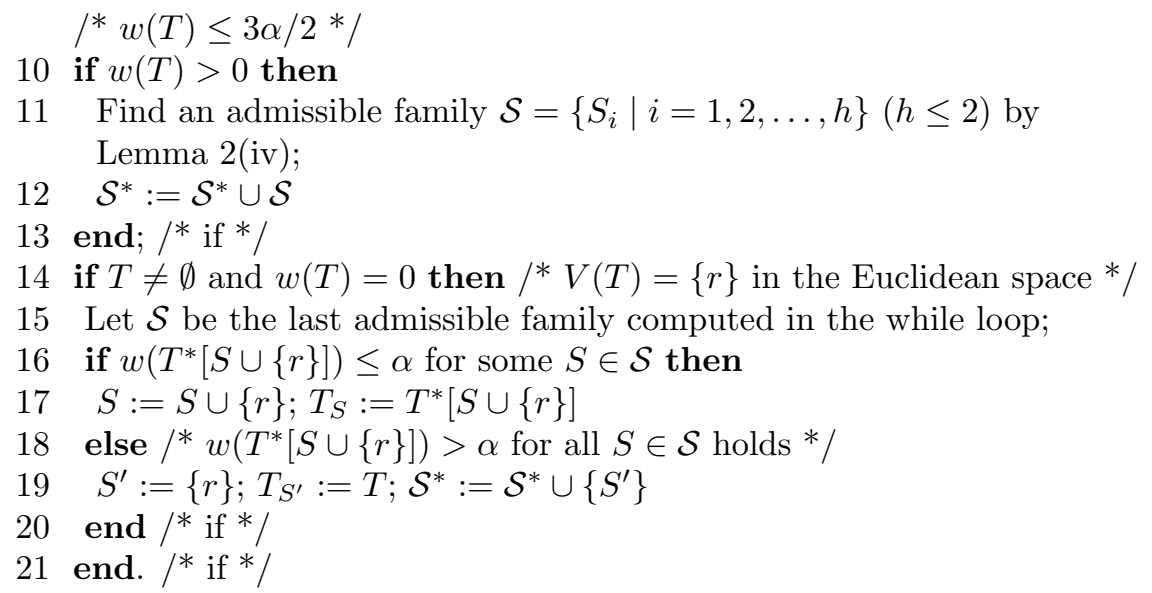

Theorem 8 Algorithm TreeCover delivers a $k$-admissible family $S^{*}$ in $T^{*}$ such that $\bigcup_{S \in S^{*}} S=V\left(T^{*}\right)$.

Proof: In each iteration of the while-loop, at least one of Theorems 5, 6, and 7 is applied to find an admissible family $\mathcal{S}$ in the current tree $T$. After the last iteration of the while-loop, we have $w(T) \leq 3 \alpha / 2$. Assume that $T=\emptyset$ or $w(T)>0$ holds for the remaining tree $T$ after line 9 . Then Lemma 2 (iv) can be applied to find another admissible family $\mathcal{S}$ for $T$ if $w(T)>0$. By Lemma 2 (i), the union of all resulting admissible families gives a desired admissible family $\mathcal{S}^{*}$ in $T^{*}$. By the definition of admissible family, the admissible family $\mathcal{S}^{*}$ contains at most $k$ subsets since $w\left(T^{*}\right)=k$.

Next assume that $T \neq \emptyset$ and $w(T)=0$ for the remaining tree $T$ after line 9 . Then $T=(\{r\}, \emptyset)$ since otherwise $w(T)$ would be positive in the Euclidean space. Let $\mathcal{S}$ be the last admissible family computed in the while loop. Now, if $w\left(T^{*}[S \cup\{r\}]\right) \leq \alpha$ holds for some $S \in \mathcal{S}$, then the new subset $S:=S \cup\{r\}$ computed in line 17 is an admissible set. Again by Lemma 2(i), the union of all resulting admissible families gives a desired admissible family $\mathcal{S}^{*}$ in $T^{*}$, and the admissible family $\mathcal{S}^{*}$ contains at most $k$ subsets since $w\left(T^{*}\right)=k$.

Assume that $w\left(T^{*}[S \cup\{r\}]\right)>\alpha$ for all $S \in \mathcal{S}$. Hence, by Theorems 5 , 6 , and 7. $\mathcal{S} \cup\left\{S^{\prime}\right\}$ is an admissible set for the subset $S^{\prime}$ computed in line 19 . Therefore, by Lemma 2(i), the union of all resulting admissible families gives a desired admissible family in $T^{*}$, and the admissible family $\mathcal{S}^{*}$ contains at most $k$ subsets since $w\left(T^{*}\right)=k$. This completes the proof.

\section{Proof of Theorem 5}

This section is devoted to present a proof of Theorem 5 which holds for any $\alpha \in[5 / 3,2]$.

For a non-root vertex $u$ in a rooted tree $T$, a partition $\left\{\mathcal{S}_{1}, \mathcal{S}_{2}, \ldots, \mathcal{S}_{p}\right.$, $\mathcal{L}, \mathcal{M}, \mathcal{H}\}$ of $\mathcal{B}(u)$ is called valid if $1 \leq w\left(\mathcal{S}_{i}\right) \leq \alpha, i=1,2, \ldots, p, \mathcal{L} \subseteq \mathcal{L}(u)$, 
354 S. Karakawa, E. Morsy, and H. Nagamochi Minmax Tree Cover in the Euclidean Space

$\mathcal{M} \subseteq \mathcal{M}(u)$, and $\mathcal{H} \subseteq \mathcal{H}(u)$. Note that, by Lemma 2(iii), $S_{i}=V\left(\mathcal{S}_{i}\right)-\{u\}$ in a valid partition forms an admissible family with a 1-admissible tree $\mathcal{S}_{i}$.

Lemma 3 Let $u \in V_{1}(T)$ be a 1-boundary vertex in a rooted tree $T$. Then there exists a valid partition $\left\{\mathcal{S}_{1}, \mathcal{S}_{2}, \ldots, \mathcal{S}_{p}, \mathcal{L}, \mathcal{M}, \mathcal{H}\right\}$ of $\mathcal{B}(u)$ that satisfies the following (i)-(ii), where $\mathcal{B}=\mathcal{L} \cup \mathcal{M} \cup \mathcal{H}$.

(i) If $\mathcal{H} \neq \emptyset$, then $\mathcal{M}=\emptyset$ and $w(\mathcal{L})<2-\alpha$ hold.

(ii) $w(\mathcal{B})<1$ holds if and only if $|\mathcal{H}| \leq 1$ holds.

Proof: We first partition $\mathcal{L}(u)$ into $\mathcal{M}_{1}^{\prime}, \mathcal{M}_{2}^{\prime}, \ldots, \mathcal{M}_{q}^{\prime}$ and $\mathcal{L}^{\prime}$ so that

$$
w\left(\mathcal{L}^{\prime}\right)<2-\alpha \quad \text { and } \quad 2-\alpha \leq w\left(\mathcal{M}_{i}^{\prime}\right) \leq \alpha-1, i=1,2, \ldots, q
$$

Since $\alpha \geq 5 / 3$ and $w(L)<2-\alpha$ for all $L \in \mathcal{L}(u)$, such a partition can be obtained by constructing $\left\{\mathcal{M}_{i}^{\prime}\right\}$ adding branches in $\mathcal{L}(u)$ one by one until the weight of the remaining branches becomes less than $2-\alpha$. We now treat each $\mathcal{M}_{i}^{\prime}$ as a single subtree $M_{i}^{\prime}$. Let $\mathcal{H}(u)=\left\{H_{1}, H_{2}, \ldots, H_{h}\right\}$ and $\mathcal{M}(u) \cup\left\{M_{i}^{\prime} \mid\right.$ $i=1,2, \ldots, q\}=\left\{M_{1}, M_{2}, \ldots, M_{m}\right\}$. Note that any pair $\left\{H_{i}, M_{j}\right\}$ gives an 1-admissible tree since $w\left(H_{i}+M_{j}\right) \geq(\alpha-1)+(2-\alpha)=1$ and $w\left(H_{i}+M_{j}\right) \leq$ $1+(\alpha-1)=\alpha$ hold.

(a) $h \geq m$. In this case, we construct $m$ sets of branches, $\mathcal{S}_{i}=\left\{H_{i}, M_{i}\right\}$, $i=1,2, \ldots, m$. If $h>m$ and $w\left(\mathcal{L}^{\prime}\right)+w\left(H_{m+1}\right) \geq 1$, then we construct $\mathcal{S}_{m+1}=$ $\mathcal{L}^{\prime} \cup\left\{H_{m+1}\right\}$, letting $p=m+1, \mathcal{L}:=\mathcal{M}:=\emptyset$, and $\mathcal{H}:=\left\{H_{i} \mid i=m+2, \ldots, h\right\}$. Otherwise, let $p=m, \mathcal{L}:=\mathcal{L}^{\prime}, \mathcal{M}:=\emptyset$, and $\mathcal{H}:=\left\{H_{i} \mid i=m+1, \ldots, h\right\}$.

(b) $h<m$. In this case, we first construct $h$ sets of branches, $\mathcal{S}_{i}=\left\{H_{i}, M_{i}\right\}$, $i=1,2, \ldots, h$. We then partition the set $\mathcal{B}^{\prime}=\mathcal{B}(u)-\cup_{1 \leq i \leq h} \mathcal{S}_{i}$ into subsets $\mathcal{S}_{j}$, $j=h+1, h+2, \ldots, p$ and $\mathcal{B}$ so that

$$
w(\mathcal{B})<1 \text { and each } \mathcal{S}_{j} \text { is a minimal subset with } w\left(\mathcal{S}_{j}\right) \geq 1 \text {. }
$$

Such a partition can be obtained by repeatedly choosing such a minimal subset $\mathcal{S}_{j}$ from $\mathcal{B}^{\prime}$ until the weight of the remaining branches becomes less than 1 . Since $w(B) \leq \alpha-1$ for all $B \in \mathcal{B}^{\prime}$, we see that $1 \leq w\left(\mathcal{S}_{j}\right) \leq 1+\alpha-1=\alpha$, i.e., $\mathcal{S}_{j}$ is 1-admissible. Let $\mathcal{L}:=\mathcal{B} \cap \mathcal{L}(u), \mathcal{M}:=\mathcal{B} \cap \mathcal{M}(u)$, and $\mathcal{H}:=\emptyset$.

Next we prove that properties (i) and (ii) hold in both cases (a) and (b).

(i) If $\mathcal{H} \neq \emptyset$, then $\mathcal{L}, \mathcal{M}$, and $\mathcal{H}$ are constructed in case (a) and thereby $\mathcal{M}=\emptyset$ and $w(\mathcal{L})<2-\alpha$ hold.

(ii) By construction, $w(\mathcal{B})<1$ holds if and only if $w(\mathcal{B})=w(\mathcal{H})+w(\mathcal{L})<1$ holds in $(\mathrm{a})$ or $w(\mathcal{B})=w(\mathcal{M})+w(\mathcal{L})<1$ holds in $(\mathrm{b})$. Since $w(H)>\alpha-1(>$ 1/2) holds for every $H \in \mathcal{H}$, we obtain $|\mathcal{H}| \leq 1$.

Given a rooted tree $T$, the following algorithm converts $T$ into another tree $T^{\prime}$ in which all 1-boundary vertices are inactive. Such a tree $T^{\prime}$ is constructed by applying a procedure that first chooses an active vertex $u \in V_{1}(T)$ in the current tree $T$, computes a valid partition $\left\{\mathcal{S}_{1}, \mathcal{S}_{2}, \ldots, \mathcal{S}_{p}, \mathcal{L}, \mathcal{M}, \mathcal{H}\right\}$ of $\mathcal{B}(u)$ by Lemma 3 and then removes the admissible family $\left\{\mathcal{S}_{1}, \mathcal{S}_{2}, \ldots, \mathcal{S}_{p}\right\}$ (if any) 
from $T$. We observe that if $u$ is a 1-boundary vertex in the resulting tree $T^{\prime}$ (i.e., $w\left(T_{e(u)}^{\prime}\right) \geq 1$ ), then either (i) $u$ is inactive if $w\left(T_{u}^{\prime}\right)=w(\mathcal{B})>\alpha$, or (ii) $w\left(T_{u}^{\prime}\right)=w(\mathcal{B}) \leq \alpha$ and $w\left(T_{e(u)}^{\prime}\right) \geq 1$ otherwise, where $\mathcal{B}=\mathcal{L} \cup \mathcal{M} \cup \mathcal{H}$. In the latter case, $D_{T^{\prime}}(u)$ is an admissible set by Lemma 2 (ii). In other words, the above procedure makes an active 1-boundary vertex inactive or creates an admissible set.

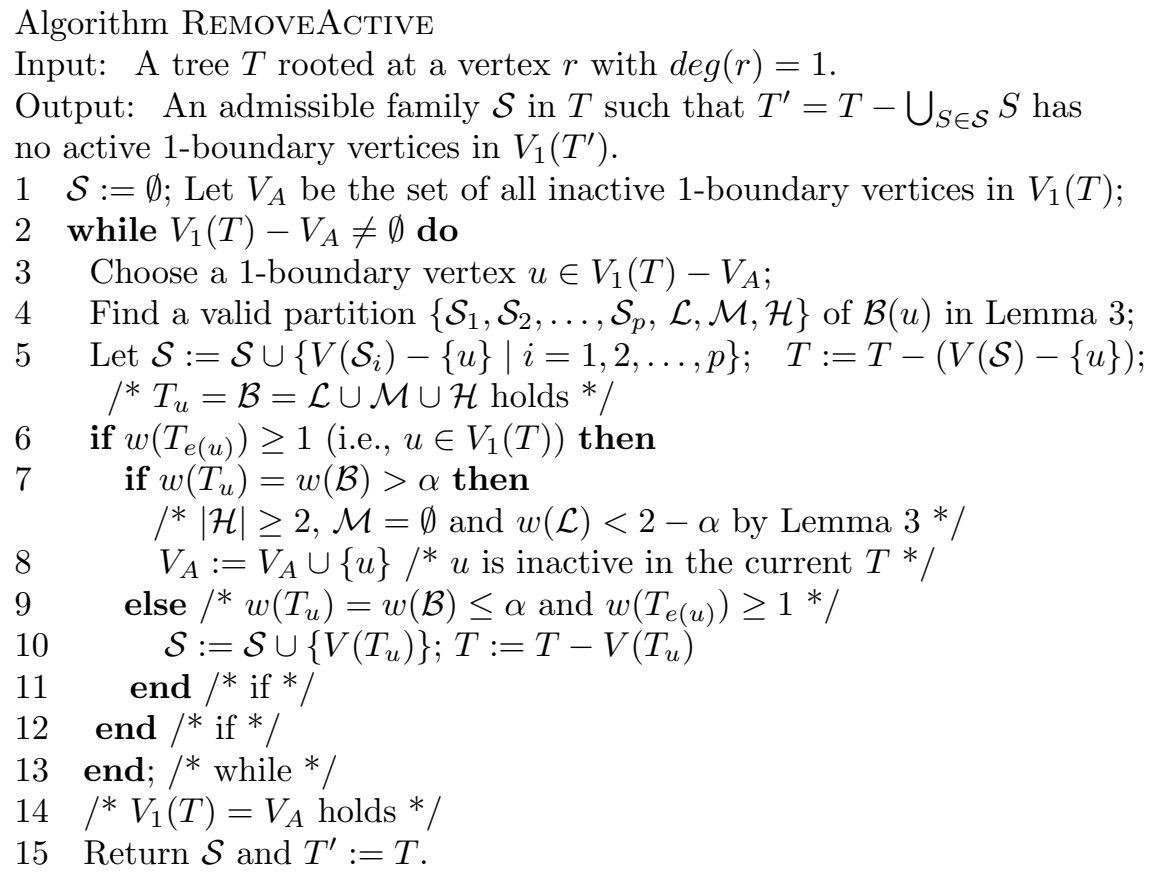

Lemma 4 Given a rooted tree T, REMOveActive outputs an admissible family $\mathcal{S}$ and a tree $T^{\prime}$ such that all vertices in $V_{1}\left(T^{\prime}\right)$ are inactive.

Proof: Note that RemoveActive terminates when $V_{1}\left(T^{\prime}\right)=V_{A}$ holds. Consider the case where a 1-boundary vertex $u \in V_{1}(T)$ in the current tree $T$ is added to $V_{A}$ during the execution of RemoveActive. We see that this $u$ satisfies Condition A, since condition $\mathrm{A}(\mathrm{i})$ holds by $w\left(T_{u}\right)=w(\mathcal{B})>\alpha$, and this implies condition $\mathrm{A}(\mathrm{ii})|\mathcal{H}(u)| \geq 2$, condition $\mathrm{A}($ iii) $\mathcal{M}(u)=\emptyset$, and condition $\mathrm{A}(\mathrm{iv}) w(\mathcal{L}(u))<2-\alpha$ by Lemma 3 . By the definition of 1-boundary vertices, no ancestor of a vertex in $V_{A}$ will be chosen in line 3 . Thus any vertex in $V_{A}$ remains to be a 1-boundary vertex upon completion of RemoveActive. This proves the lemma.

Lemma 5 For the admissible family $\mathcal{S}$ and the tree $T^{\prime}$ output from REMOVEACTIVE, if $T^{\prime} \neq \emptyset, w\left(T^{\prime}\right)=0$, and $w(T[S \cup\{r\}])>\alpha$ for all $S \in \mathcal{S}$, then $\mathcal{S} \cup V\left(T^{\prime}\right)$ is an admissible family in $T$.

Proof: Let $T_{0}$ denote the tree input to RemoveActive. Assume that $T^{\prime} \neq \emptyset$, $w\left(T^{\prime}\right)=0$, and $w(T[S \cup\{r\}])>\alpha$ for all $S \in \mathcal{S}$. Note that $T^{\prime} \neq \emptyset$ and $w\left(T^{\prime}\right)=0$ 
356 S. Karakawa, E. Morsy, and H. Nagamochi Minmax Tree Cover in the Euclidean Space

imply that $T^{\prime}=(\{r\}, \emptyset)$ in the Euclidean space. Moreover, $T^{\prime}=(\{r\}, \emptyset)$ is obtained in lines 9-10, where applied to a subtree $T_{u}$ for a 1-boundary vertex $u$ incident to $r$. Clearly $w\left(T_{u}\right)<\alpha$ and $w\left(T^{\prime}\right)=0<\alpha$. Note that $\mathcal{S}-\left\{V\left(T_{u}\right)\right\}$ is also an admissible family in $T$ by Lemma 3 . Hence there are trees $T_{S}$ with $S \subseteq V\left(T_{S}\right)$ and $w\left(T_{S}\right) \leq \alpha$ for all $S \in \mathcal{S}-\left\{V\left(T_{u}\right)\right\}$, and the subtree $T\left[V\left(T_{u}\right) \cup\right.$ $\{r\}]=T_{0}-\cup_{S \in \mathcal{S}-\left\{V\left(T_{u}\right)\right\}} S$ satisfies $w\left(T_{0}\right)-w\left(T\left[V\left(T_{u}\right) \cup\{r\}\right]\right) \geq|\mathcal{S}|-1$. By the assumption on the lemma, $w\left(T\left[V\left(T_{u}\right) \cup\{r\}\right]\right)>\alpha>1$ holds. Hence we have $w\left(T_{0}\right) \geq|\mathcal{S}|-1+w\left(T\left[V\left(T_{u}\right) \cup\{r\}\right]\right)>|\mathcal{S}|$, and thereby $\left\lceil w\left(T_{0}\right)\right\rceil \geq|\mathcal{S}|+1=$ $\left|\mathcal{S} \cup\left\{V\left(T^{\prime}\right)\right\}\right|$. Then we conclude that $\mathcal{S} \cup\left\{V\left(T^{\prime}\right)\right\}$ is an admissible family in $T$. This proves the lemma.

Theorem 5 follows from Lemmas 4 and 5 .

\section{Proof of Theorem 6}

This section gives a proof of Theorem 6 . For this, we assume that $\alpha=2 \sqrt{3}-3 / 2$ throughout this section.

For a rooted tree $T$, consider a branch $T_{e}$ of a vertex $u$ in $T$ such that $2 \alpha-3 \leq w\left(T_{e}\right)<1$ and $T_{e(u)} \geq 1$. We denote by $\mathcal{H}^{*}(T)$ the set of such branches $T_{e}$ in $T$.

Lemma 6 Let $T$ be a rooted tree which has no active 1-boundary vertices in $V_{1}(T)$. Then every 2-boundary vertex $z \in V_{2}(T)$ satisfies the following condition. Condition B:

(i) $\left|\mathcal{H}^{*}\left(T_{z}\right)\right| \geq 2$, (ii) $|\mathcal{H}(u)|=2$ for all $u \in V_{1}\left(T_{z}\right)$, (iii) $w(z, u)<2-\alpha$ for all $u \in V_{1}\left(T_{z}\right)$.

Proof: Let $z$ be an arbitrary 2-boundary vertex $z \in V_{2}(T)$.

Tree $T_{z}$ contains a vertex $u$ that is a 1-boundary vertex in $T$. If $V_{1}\left(T_{z}\right) \neq \emptyset$, then this is true; otherwise $\left(V_{1}\left(T_{z}\right)=\emptyset\right) z$ is a 1-boundary vertex in $T$. Let $u$ be a 1-boundary vertex $u \in V_{1}(T) \cap V\left(T_{z}\right)$. Then $|\mathcal{H}(u)| \geq 2$ holds by condition A(ii), and $u$ has two branches $T_{e}$ and $T_{e^{\prime}}$ with $w\left(T_{e}\right), w\left(T_{e^{\prime}}\right) \in(\alpha-1,1) \subseteq(2 \alpha-3,1)$ by $\alpha \leq 2$. We see that $T_{e}, T_{e^{\prime}} \in \mathcal{H}^{*}\left(T_{z}\right)$ since $u$ is a 1-boundary vertex and $w\left(T_{e(u)}\right) \geq 1$ holds. Hence $\left|\mathcal{H}^{*}\left(T_{z}\right)\right| \geq 2$ holds, proving $\mathrm{B}(\mathrm{i})$.

By Condition A(ii), $|\mathcal{H}(u)| \geq 2$ holds for all 1-boundary vertices $u \in V_{1}\left(T_{z}\right) \subseteq$ $V_{1}(T)$. If $|\mathcal{H}(u)| \geq 3$ for some $u \in V_{1}\left(T_{z}\right)$, then we would have

$$
w\left(T_{u}\right)>3(\alpha-1) \geq 2
$$

since $\alpha \geq 5 / 3$, contradicting that $z \in V_{2}(T)$. Hence $|\mathcal{H}(u)|=2$ holds for all $u \in V_{1}\left(T_{z}\right)$, i.e., $\mathrm{B}(\mathrm{ii})$ holds.

Finally, we prove $\mathrm{B}(\mathrm{iii})$. Let $T_{e} \in \mathcal{B}(z)$ satisfy $u \in V_{1}\left(T_{e}\right)$, and $P_{T}(z, u)$ be the path between $z$ and $u$ along $T$. Since $T_{e}$ is a branch of a 2-boundary vertex $z$ of $T, w\left(T_{e}\right)<2$ holds. On the other hand, by condition $\mathrm{A}(\mathrm{i}), w\left(T_{u}\right)>\alpha$ holds. Therefore we have

$$
w\left(P_{T}(z, u)\right) \leq w\left(T_{e}\right)-w\left(T_{u}\right)<2-\alpha .
$$


Since $(G, w)$ is a metric, we have $w(z, u) \leq w\left(P_{T}(z, u)\right)<2-\alpha$.

Note that, for any $T^{\prime} \in \mathcal{H}^{*}\left(T_{z}\right)$ with a root $r^{\prime}$, the root $r^{\prime}$ must be $z$ or a 1-boundary vertex in $V_{1}\left(T_{z}\right)$ since $w\left(T_{e\left(r^{\prime}\right)}\right) \geq 1$ must hold by the maximality of $T^{\prime}$.

We distinguish two cases; (i) $\left|\mathcal{H}^{*}\left(T_{z}\right)\right|=2$ and (ii) $\left|\mathcal{H}^{*}\left(T_{z}\right)\right| \geq 3$. In case (i), we find a 2 -admissible family in $T_{z}$. In case (ii), we find a 1-admissible family $\{S\}$, where the corresponding 1-admissible tree $T_{S}$ will be constructed by introducing an edge which is not in tree $T$.

\subsection{Case of $\left|\mathcal{H}^{*}\left(T_{z}\right)\right|=2$}

We first consider the case where $\left|\mathcal{H}^{*}\left(T_{z}\right)\right|=2$.

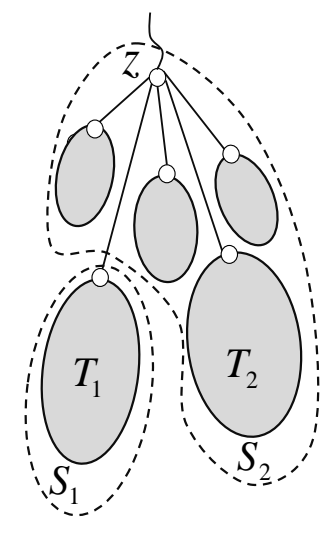

(a)

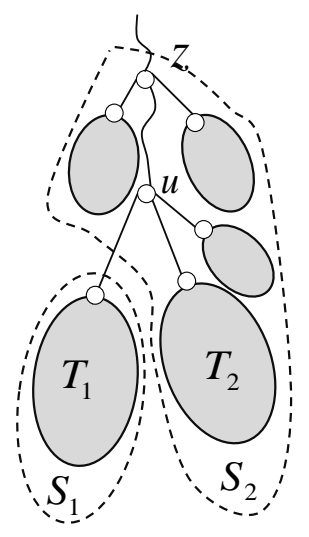

(b)

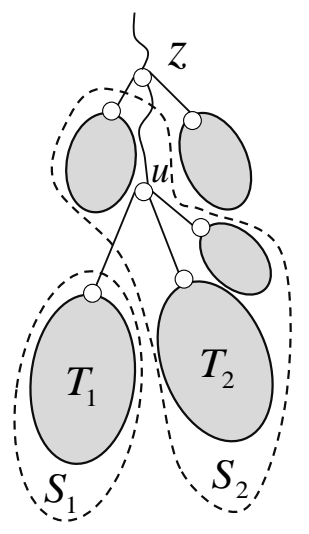

(c)

Figure 1: Illustration of Lemma 7, where (a), (b), and (c) represent (i), (ii), and (iii), respectively.

Lemma 7 Let $T$ be a rooted tree which has no active 1-boundary vertex, and let $z \in V_{2}(T)$ satisfy $\left|\mathcal{H}^{*}\left(T_{z}\right)\right|=2$. Then $\left|V_{1}(T) \cap V\left(T_{z}\right)\right|=1$. Furthermore, for $\mathcal{H}^{*}\left(T_{z}\right)=\left\{T_{1}, T_{2}\right\}$, one of the following holds:

(i) If $V_{1}(T) \cap V\left(T_{z}\right)=\{z\}$ holds, then $S_{1}:=V\left(T_{1}\right)$ and $S_{2}:=V\left(T_{z}\right)-S_{1}$ give a 2-admissible family in $T$ (see Fig. 11(a)).

(ii) If $V_{1}(T) \cap V\left(T_{z}\right)=\{u\}, u \neq z$, and $w\left(T_{z}\right)<2$ hold, then $S_{1}:=V\left(T_{1}\right)$ and $S_{2}:=V\left(T_{z}\right)-S_{1}$ give a 2-admissible family in $T$ (see Fig. 1. (b)). 
358 S. Karakawa, E. Morsy, and H. Nagamochi Minmax Tree Cover in the Euclidean Space

(iii) If $V_{1}(T) \cap V\left(T_{z}\right)=\{u\}, u \neq z$, and $w\left(T_{z}\right) \geq 2$ hold, then for any minimal subset $\mathcal{S} \subseteq \mathcal{B}(z)-\left\{T_{e}\right\}$ satisfying $w\left(T_{e}\right)+w(\mathcal{S}) \geq 2, S_{1}:=V\left(T_{1}\right)$ and $S_{2}:=V\left(T_{e}\right) \cup V(\mathcal{S})-\left(S_{1} \cup\{z\}\right)$ give a 2-admissible family in $T$, where $T_{e} \in \mathcal{B}(z)$ satisfies $u \in V\left(T_{e}\right)$ (see Fig. 1 (c)).

Proof: We first show that $\left|V_{1}(T) \cap V\left(T_{z}\right)\right|=1$. If $z \in V_{1}(T) \cap V\left(T_{z}\right)$, then no descendant of $z$ is a 1-boundary vertex in $T$ since $w\left(T_{e(v)}\right)<1$ for all $v \in$ $C h(z)$, i.e., $V_{1}(T) \cap V\left(T_{z}\right)=\{z\}$. Assume that $z \notin V_{1}(T) \cap V\left(T_{z}\right)$. Since $w\left(T_{e(z)}\right) \geq 2$ holds by $z \in V_{2}(T)$, a descendant $u$ of $z$ must be a 1 -boundary vertex in $T$ (also in $T_{z}$ ). Such a vertex $u \in V_{1}\left(T_{z}\right)$ has two children $v$ and $v^{\prime}$ with $w\left(T_{e(v)}\right), w\left(T_{e\left(v^{\prime}\right)}\right)>\alpha-1 \geq 2 \alpha-3$, since $|\mathcal{H}(u)|=2$ holds by Condition B(ii), implying that $w\left(T_{e(v)}\right), w\left(T_{e\left(v^{\prime}\right)}\right) \in \mathcal{H}^{*}\left(T_{z}\right)$ (note that $w\left(T_{e(v)}\right), w\left(T_{e\left(v^{\prime}\right)}\right)<1$ by $u \in V_{1}(T)$ ). Therefore, by $\left|\mathcal{H}^{*}\left(T_{z}\right)\right|=2$, such a vertex $u$ is unique. Hence one of the following holds: (i) $V_{1}(T) \cap V\left(T_{z}\right)=\{z\}$, (ii) $V_{1}(T) \cap V\left(T_{z}\right)=\{u\}, u \neq z$, and $w\left(T_{z}\right)<2$, and (iii) $V_{1}(T) \cap V\left(T_{z}\right)=\{u\}, u \neq z$, and $w\left(T_{z}\right) \geq 2$.

(i) Since $z \in V_{1}(T)$, by Condition $\mathrm{A}($ iii)-(iv), we have a partition $\mathcal{B}(z)=$ $\left\{T_{1}, T_{2}\right\} \cup \mathcal{L}(z)$. Then $T_{S_{1}}:=T_{1}$ and $T_{S_{2}}:=T\left[S_{2} \cup\{z\}\right]$ satisfy

$$
\begin{aligned}
& w\left(T_{S_{1}}\right)=w\left(T_{1}\right)<1 \\
& w\left(T_{S_{2}}\right)=w\left(T_{2}\right)+w(\mathcal{L}(z))<1+(2-\alpha)=3-\alpha<\alpha .
\end{aligned}
$$

Furthermore, for $T^{\prime}:=T-\left(S_{1} \cup S_{2}\right), w(T)-w\left(T^{\prime}\right) \geq 2$ holds by $z \in V_{2}(T)$. This implies that $\left\{S_{1}, S_{2}\right\}$ is 2-admissible.

(ii) Note that $u \in V_{1}\left(T_{z}\right)$ is unique and $\mathcal{H}(u) \cap \mathcal{H}^{*}\left(T_{z}\right)=\left\{T_{1}, T_{2}\right\}$ holds by Condition B(ii). Then $T_{S_{1}}:=T_{1}$ and $T_{S_{2}}:=T\left[S_{2} \cup\{u\}\right]$ satisfy

$$
\begin{aligned}
& w\left(T_{S_{1}}\right)=w\left(T_{1}\right)<1 \\
& w\left(T_{S_{2}}\right)=w\left(T_{z}\right)-w\left(T_{1}\right)<2-(\alpha-1)=3-\alpha<\alpha .
\end{aligned}
$$

Since $z \in V_{2}(T)$ holds, we have $w(T)-w\left(T^{\prime}\right) \geq 2$ for $T^{\prime}:=T-\left(S_{1} \cup S_{2}\right)$. Therefore $\left\{S_{1}, S_{2}\right\}$ is 2-admissible.

(iii) Since $w\left(T_{z}\right)=w\left(T_{e}\right)+w\left(\mathcal{B}(z)-\left\{T_{e}\right\}\right) \geq 2$, there exists a minimal subset $\mathcal{S} \subseteq \mathcal{B}(z)-\left\{T_{e}\right\}$ such that $w\left(T_{e}\right)+w(\mathcal{S}) \geq 2$ holds. Then the minimality of $\mathcal{S}$ implies

$$
2 \leq w\left(T_{e}\right)+w(\mathcal{S}) \leq 2+(2 \alpha-3)=2 \alpha-1,
$$

because for every $B \in \mathcal{S}, w(B)<2 \alpha-3$ holds. Since $\mathcal{H}(u)=\left\{T_{1}, T_{2}\right\}$ holds (i.e., $w\left(T_{1}\right)>\alpha-1$ holds), $T_{S_{1}}:=T_{1}$ and $T_{S_{2}}:=T\left[S_{2} \cup\{u, z\}\right]$ satisfy

$$
\begin{aligned}
w\left(T_{S_{1}}\right) & =w\left(T_{1}\right)<1 \\
w\left(T_{S_{2}}\right) & =w\left(T_{e}\right)+w(\mathcal{S})-w\left(T_{1}\right) \\
& <(2 \alpha-1)-(\alpha-1)=\alpha .
\end{aligned}
$$

Furthermore, $w\left(T_{S_{1}}\right)+w\left(T_{S_{2}}\right)=w\left(T_{e}\right)+w(\mathcal{S}) \geq 2$ holds, implying that $\left\{S_{1}, S_{2}\right\}$ is 2-admissible. 


\subsection{Case of $\left|\mathcal{H}^{*}\left(T_{z}\right)\right| \geq 3$}

We next consider the case where $\left|\mathcal{H}^{*}\left(T_{z}\right)\right| \geq 3$. In this case, we construct a 1-admissible tree by introducing an edge not in the current tree $T$.

The next lemma describes a property of the angle formed by two of three line segments in the Euclidean space $\mathbb{R}^{d}$.

Lemma 8 For vertices $z, v_{1}, v_{2}$, and $v_{3}$, the minimum angle $\theta$ formed by line segments $\overline{z v_{i}}$ and $\overline{z v_{j}}, i \neq j, i, j=1,2,3$, is no more than $120^{\circ}$.

Proof: Let $\gamma$ be the plane that contains three points $v_{1}, v_{2}, v_{3}$. Without loss of generality, we assume that $z$ is on the origin 0 . We distinguish two cases; (i) $z$ is also on the plane $\gamma$, and (ii) $z$ is not on the plane $\gamma$.

(i) The origin 0 is on $\gamma$. Then, obviously, three line segments $\left(0, v_{1}\right),\left(0, v_{2}\right),\left(0, v_{3}\right)$ divide $360^{\circ}$ to three and hence the minimum angle $\theta$ formed by two of such segments is no more than $120^{\circ}$.

(ii) The origin 0 is not on $\gamma$. Let $x$ be the projection of 0 to $\gamma$. We have the following two situations.

(a) $x$ is equal to one of the points $v_{1}, v_{2}, v_{3}$; assume without loss of generality that $x=v_{1}$. Since $v_{1}$ is the projection of 0 to $\gamma$, the vector $v_{1}$ intersects vectors $v_{2}-v_{1}$ and $v_{3}-v_{1}$ orthogonally; i.e.,

$$
v_{1} \cdot\left(v_{2}-v_{1}\right)=v_{1} \cdot\left(v_{3}-v_{1}\right)=0
$$

where $u \cdot v$ is the inner product of $u, v \in \mathbb{R}^{d}$. From this, we obtain

$$
v_{1} \cdot v_{2}=v_{1} \cdot v_{3}=v_{1} \cdot v_{1}=\left\|v_{1}\right\|^{2} .
$$

Let $\varphi$ be the angle formed by $\left(0, v_{1}\right)$ and $\left(0, v_{2}\right)$. Then we have

$$
\cos \varphi=\frac{v_{1} \cdot v_{2}}{\left\|v_{1}\right\|\left\|v_{2}\right\|}=\frac{\left\|v_{1}\right\|^{2}}{\left\|v_{1}\right\|\left\|v_{2}\right\|}=\frac{\left\|v_{1}\right\|}{\left\|v_{2}\right\|}>0 .
$$

This implies that the minimum angle $\theta$ is no more than $120^{\circ}$.

(b) $x$ is not equal to $v_{1}, v_{2}$ and $v_{3}$. Let $\psi$ be the minimum angle formed by two of $\left(x, v_{1}\right),\left(x, v_{2}\right),\left(x, v_{3}\right)$; assume without loss of generality that $\psi$ is formed by $\left(x, v_{1}\right)$ and $\left(x, v_{2}\right)$. Then $\psi \leq 120^{\circ}$ holds by (i) since $x, v_{1}, v_{2}, v_{3}$ are on $\gamma$. Since $x$ is the projection to $\gamma$, the vector $x$ intersects vectors $v_{1}-x, v_{2}-x$ and $v_{3}-x$ orthogonally; i.e.,

$$
x \cdot\left(v_{1}-x\right)=x \cdot\left(v_{2}-x\right)=x \cdot\left(v_{3}-x\right)=0 .
$$

From this, we obtain

$$
x \cdot v_{1}=x \cdot v_{2}=x \cdot v_{3}=x \cdot x=\|x\|^{2} .
$$

Then we have

$$
\cos \psi=\frac{\left(x-v_{1}\right) \cdot\left(x-v_{2}\right)}{\left\|x-v_{1}\right\|\left\|x-v_{2}\right\|} \geq-\frac{1}{2} .
$$


360 S. Karakawa, E. Morsy, and H. Nagamochi Minmax Tree Cover in the Euclidean Space

Therefore, we conclude that

$$
v_{1} \cdot v_{2} \geq\|x\|^{2}-\frac{1}{2}\left\|x-v_{1}\right\|\left\|x-v_{2}\right\|
$$

Now, let $\varphi$ be the angle formed by $\left(0, v_{1}\right)$ and $\left(0, v_{2}\right)$. Then $\varphi$ satisfies

$$
\cos \varphi=\frac{v_{1} \cdot v_{2}}{\left\|v_{1}\right\|\left\|v_{2}\right\|} \geq \frac{\|x\|^{2}}{\left\|v_{1}\right\|\left\|v_{2}\right\|}-\frac{\left\|x-v_{1}\right\|\left\|x-v_{2}\right\|}{2\left\|v_{1}\right\|\left\|v_{2}\right\|} .
$$

Note that $\left\|x-v_{1}\right\|^{2}=\left(x-v_{1}\right) \cdot\left(x-v_{1}\right)=\left\|v_{1}\right\|^{2}-\|x\|^{2}\left(\right.$ since $\left.x \cdot v_{1}=\|x\|^{2}\right)$. Therefore, $\left\|x-v_{1}\right\|<\left\|v_{1}\right\|$ since $\|x\|,\left\|v_{1}\right\|,\left\|x-v_{1}\right\|>0$. Similarly, we can conclude that $\left\|x-v_{2}\right\|<\left\|v_{2}\right\|$. Hence, we have

$$
\cos \varphi>\frac{\|x\|^{2}}{\left\|v_{1}\right\|\left\|v_{2}\right\|}-\frac{1}{2}>-\frac{1}{2} .
$$

Therefore, we obtain $\varphi \leq 120^{\circ}$.

This implies that the minimum angle $\theta$ is no more than $120^{\circ}$ in all situations.

For a descendent $v$ of a vertex $u$ in a rooted tree $T$, the $(1 / 2)$-distant point of $v$ with $u$ is defined by the point $p_{u v}$ on the line segment $u v$ such that $w\left(u, p_{u v}\right)=$ $\min \{1 / 2, w(u, v)\}$; i.e., $p_{u v}=v$ if $w(u, v) \leq 1 / 2$, and $p_{u v}$ is the point on $u v$ satisfying $w\left(u, p_{u v}\right)=1 / 2$ otherwise.

Let $z$ be a 2-boundary vertex in a rooted tree $T$ which has no active 1boundary vertices. By definition, the root $u_{i}$ of any branch $T_{i} \in \mathcal{H}^{*}\left(T_{z}\right)$ is a 1-boundary vertex in $T$. Therefore, the intersection of any two branches $T_{i}, T_{j} \in \mathcal{H}^{*}\left(T_{z}\right)$ contains a vertex if and only if they have the same root, i.e., $u_{i}=u_{j}$. We also note that a $1 / 2$-boundary vertex $v_{i} \in V_{1 / 2}\left(T_{i}\right)$ is unique since $w\left(T_{i}\right)<1$.

The next lemma claims that if $\left|\mathcal{H}^{*}\left(T_{z}\right)\right| \geq 3$, then an admissible set $\mathcal{S}$ can be found or $\mathcal{H}^{*}\left(T_{z}\right)$ contains a pair of branches $T_{i}$ and $T_{j}$ that satisfies a certain condition.

Lemma 9 Let $T$ be a rooted tree which has no active 1-boundary vertices in $V_{1}(T)$. Let $z$ be a 2-boundary vertex in $V_{2}(T)$ such that there are three subtrees $T_{1}, T_{2}, T_{3} \in \mathcal{H}^{*}\left(T_{z}\right)$, where $u_{i}$ denotes the root of $T_{i}\left(u_{i} \in V_{1}(T) \cap V\left(T_{z}\right)\right)$, $i=1,2,3$. For each $i=1,2,3$, let $v_{i}$ be a 1/2-boundary vertex in $V_{1 / 2}\left(T_{i}\right)$. Then:

(a) For each $T_{i} \in\left\{T_{1}, T_{2}, T_{3}\right\}$, there exists a branch $\tilde{T}_{i} \in \mathcal{H}\left(\tilde{u}_{i}\right) \cap \mathcal{H}^{*}\left(T_{z}\right)-T_{i}$ such that the root $\tilde{u}_{i} \in V_{1}(T) \cap V\left(T_{z}\right)$ of $\tilde{T}_{i}$ satisfies $w\left(u_{i}, \tilde{u}_{i}\right)<2-\alpha$.

(b) Assume that there is $i \in\{1,2,3\}$ such that $w\left(T_{v_{i}}\right) \geq 1 / 2$ holds. If there exists a minimal subtree $\mathcal{S} \subseteq \mathcal{B}\left(v_{i}\right)$ such that $w(\mathcal{S})+w\left(\tilde{T}_{i}\right) \geq 1$ and

$$
w(\mathcal{S})+w\left(\tilde{T}_{i}\right)+w\left(v_{i}, \tilde{u}_{i}\right) \leq \alpha,
$$

then $S:=\left(V\left(\tilde{T}_{i}\right)-\left\{\tilde{u}_{i}\right\}\right) \cup\left(V(\mathcal{S})-\left\{v_{i}\right\}\right)$ is admissible. Testing whether such a subtree exists or not can be done in $O\left(\left|\mathcal{B}\left(v_{i}\right)\right|\right)$ time. 
(c) If the condition of (b) does not hold, then there exists $\left\{T_{i}, T_{j}\right\} \subseteq\left\{T_{1}, T_{2}, T_{3}\right\}$ ( $i \neq$ $j)$ such that the tuple $\left(T, z, T_{i}, T_{j}, u_{i}, u_{j}, p_{u_{i} v_{i}}, p_{u_{j} v_{j}}\right)$ satisfies the following condition; $i=1$ and $j=2$ are assumed without loss of generality.

\section{Condition C:}

(i) For each $i=1,2,1 / 2 \leq w\left(T_{i}\right)<1$ holds.

(ii) $\theta:=\angle p_{u_{1} v_{1}} z p_{u_{2} v_{2}} \leq 120^{\circ}$.

(iii) For each $i=1,2, w\left(z, u_{i}\right)<2-\alpha$ holds.

(iv) For each $i=1,2$, if $w\left(T_{v_{i}}\right) \geq 1 / 2$ holds, then $\overline{\mathcal{B}}_{i}:=\mathcal{B}\left(v_{i}\right)-\left\{B_{i}\right\}$ satisfies $w\left(\overline{\mathcal{B}}_{i}\right)<2-\alpha$, where $B_{i} \in \mathcal{B}\left(v_{i}\right)$ is the heaviest branch of $v_{i}$.

Proof: (a) We distinguish two cases; (1) $u_{i}=z$ and $(2) u_{i} \neq z$.

(1) If $V_{1}(T) \cap V\left(T_{z}\right)=\{z\}$, then $\mathcal{H}(z)=\mathcal{H}^{*}\left(T_{z}\right)$ holds, and hence $\left|\mathcal{H}^{*}\left(T_{z}\right)\right| \geq 3$ implies that, for $\tilde{u}_{i}=z$, there is a branch $\tilde{T}_{i} \in \mathcal{H}^{*}\left(T_{z}\right)$ other than $T_{i}$, where $w\left(u_{i}, \tilde{u}_{i}\right)=w(z, z)=0$. Otherwise, there exists $\tilde{u}_{i} \in V_{1}(T) \cap V\left(T_{z}\right)$ such that $w\left(z, \tilde{u}_{i}\right)<2-\alpha$ by Condition $\mathrm{B}(\mathrm{iii})$. Then by Condition $\mathrm{B}(\mathrm{ii})$, there exists $\tilde{T}_{i} \in \mathcal{H}\left(\tilde{u}_{i}\right) \cap \mathcal{H}^{*}\left(T_{z}\right)$ such that $w\left(u_{i}, \tilde{u}_{i}\right)=w\left(z, \tilde{u}_{i}\right)<2-\alpha$ holds.

(2) By $u_{i} \neq z$, we have $T_{i} \in \mathcal{H}^{*}\left(T_{z}\right)-\mathcal{H}(z)$. This implies that $u_{i} \in V_{1}\left(T_{z}\right)$. Then by Condition B(ii), we can choose $\tilde{u}_{i}=u_{i}$ and $\tilde{T}_{i} \in \mathcal{H}\left(u_{i}\right) \cap \mathcal{H}^{*}\left(T_{z}\right)-\left\{T_{i}\right\}$, where $w\left(u_{i}, \tilde{u}_{i}\right)=w\left(u_{i}, u_{i}\right)=0$.

(b) Assume that $w\left(T_{v_{i}}\right) \geq 1 / 2$ holds for some $i \in\{1,2,3\}$. Obviously any minimal subtree $\mathcal{S} \subseteq \mathcal{B}\left(v_{i}\right)$ that satisfies $w(\mathcal{S})+w\left(\tilde{T}_{i}\right) \geq 1$ and 11 is admissible. We show that we can test whether a minimal subtree $\mathcal{S} \subseteq \mathcal{B}\left(v_{i}\right)$ satisfying $w(\mathcal{S})+w\left(\tilde{T}_{i}\right) \geq 1$ and $(1)$ exists or not in $O\left(\left|\mathcal{B}\left(v_{i}\right)\right|\right)$ time. For $v_{i} \in\left\{v_{1}, v_{2}, v_{3}\right\}$ satisfying $w\left(T_{v_{i}}\right) \geq 1 / 2$, we partition $\mathcal{B}\left(v_{i}\right)$ into the following two subsets of branches:

$$
\begin{aligned}
\mathcal{H}_{i} & :=\left\{B \in \mathcal{B}\left(v_{i}\right) \mid 4-2 \alpha<w(B)<1 / 2\right\}, \\
\mathcal{L}_{i} & :=\left\{B \in \mathcal{B}\left(v_{i}\right) \mid w(B) \leq 4-2 \alpha\right\},
\end{aligned}
$$

where $w(B)+w\left(\tilde{T}_{i}\right)>(4-2 \alpha)+(\alpha-1)>1$ holds for every $B \in \mathcal{H}_{i}$. We can test whether $w(B)+w\left(\tilde{T}_{i}\right)+w\left(v_{i}, \tilde{u}_{i}\right) \leq \alpha$ holds or not in $O\left(\left|\mathcal{H}_{i}\right|\right)$ time.

If $w\left(\mathcal{L}_{i}\right) \geq 2-\alpha$ holds, then we choose a minimal subtree $\mathcal{L}^{\prime} \subseteq \mathcal{L}_{i}$ satisfying $w\left(\mathcal{L}^{\prime}\right)+w\left(\tilde{T}_{i}\right) \geq 1$. This can be done in $O\left(\left|\mathcal{L}^{\prime}\right|\right)$ time. Therefore testing whether there exists a minimal subtree satisfying $w(\mathcal{S})+w\left(\tilde{T}_{i}\right) \geq 1$ such that 11 holds or not can be done in $O\left(\left|\mathcal{H}_{i}\right|+\left|\mathcal{L}^{\prime}\right|\right)=O\left(\left|\mathcal{B}\left(v_{i}\right)\right|\right)$ time.

We here show that $\mathcal{L}^{\prime}$ always satisfies $(1)$. By the minimality of $\mathcal{L}^{\prime}$, we have

$$
1 \leq w\left(\mathcal{L}^{\prime}\right)+w\left(\tilde{T}_{i}\right)<1+(4-2 \alpha)=5-2 \alpha .
$$

Note that $w\left(u_{i}, v_{i}\right)<1 / 2$ holds because $1>w\left(T_{i}\right)_{\tilde{T}_{i}} \geq w\left(u_{i}, v_{i}\right)+w\left(T_{v_{i}}\right) \geq$ $w\left(u_{i}, v_{i}\right)+1 / 2$ holds. By (a), there exists a branch $\tilde{T}_{i} \in \mathcal{H}\left(\tilde{u}_{i}\right) \cap \mathcal{H}^{*}\left(T_{z}\right)$ such 
362 S. Karakawa, E. Morsy, and H. Nagamochi Minmax Tree Cover in the Euclidean Space

that the root $\tilde{u}_{i} \in V_{1}(T) \cap V\left(T_{z}\right)$ of $\tilde{T}_{i}$ satisfies $w\left(u_{i}, \tilde{u}_{i}\right)<2-\alpha$. Then we have

$$
\begin{aligned}
w\left(\mathcal{L}^{\prime}\right)+w\left(\tilde{T}_{i}\right)+w\left(v_{i}, \tilde{u}_{i}\right) & <(5-2 \alpha)+w\left(u_{i}, v_{i}\right)+w\left(u_{i}, \tilde{u}_{i}\right) \\
& <(5-2 \alpha)+1 / 2+(2-\alpha) \\
& =15 / 2-3 \alpha \\
& <\alpha .
\end{aligned}
$$

Therefore $\mathcal{L}^{\prime}$ satisfies (1).

(c) By Lemma 8, we can assume that $\angle p_{u_{1} v_{1}} z p_{u_{2} v_{2}} \leq 120^{\circ}$ holds (see Fig. 2(c)). Then by assumption, $\left(T, z, T_{1}, T_{2}, u_{1}, u_{2}, p_{u_{1} v_{1}}, p_{u_{1} v_{2}}\right)$ satisfies Condition $\mathrm{C}(\mathrm{i})$ and $\mathrm{C}(\mathrm{ii})$. By $u_{1}, u_{2} \in V_{1}(T) \cap V\left(T_{z}\right)$ and Condition $\mathrm{B}($ iii), we have $w\left(z, u_{i}\right)<2-\alpha, i=1,2$, implying that Condition $\mathrm{C}($ iii) holds. Therefore it remains to show that the tuple satisfies Condition $\mathrm{C}(\mathrm{iv})$ if (b) does not hold.

Suppose that $w\left(T_{v_{i}}\right) \geq 1 / 2$ holds for some $i \in\{1,2\}$ and $B_{i}$ is the branch in $\mathcal{B}\left(v_{i}\right)$ with the maximum weight. Then $\left|\mathcal{B}\left(v_{i}\right)\right| \geq 2$ holds because of the definition of $1 / 2$-boundary vertices and $w\left(T_{v_{i}}\right) \geq 1 / 2$. This implies that $\overline{\mathcal{B}_{i}}:=$ $\mathcal{B}\left(v_{i}\right)-\left\{B_{i}\right\} \neq \emptyset$ holds, and therefore $w\left(\overline{\mathcal{B}_{i}}\right)>0$ holds. In addition, we have $w\left(B_{i}\right)<1 / 2$ because $B_{i} \in \mathcal{B}\left(v_{i}\right)$ holds for $v_{i} \in V_{1 / 2}\left(T_{i}\right) \subseteq V_{1 / 2}\left(T_{z}\right)$.

We show that $w\left(\overline{\mathcal{B}_{i}}\right)<2-\alpha$. Assume that $w\left(\overline{\mathcal{B}_{i}}\right) \geq 2-\alpha$ holds. Then by the proof of (b) and the assumption, $w\left(\mathcal{L}_{i} \cap \overline{\mathcal{B}_{i}}\right)<2-\alpha$ must hold. Then we obtain

$$
2-\alpha \leq w\left(\overline{\mathcal{B}_{i}}\right)=w\left(\mathcal{H}_{i} \cap \overline{\mathcal{B}_{i}}\right)+w\left(\mathcal{L}_{i} \cap \overline{\mathcal{B}_{i}}\right)<w\left(\mathcal{H}_{i} \cap \overline{\mathcal{B}_{i}}\right)+2-\alpha
$$

implying that $\left|\mathcal{H}_{i} \cap \overline{\mathcal{B}_{i}}\right|>0$ holds.

By assumption, every $B \in \mathcal{H}_{i} \cap \overline{\mathcal{B}_{i}}$ satisfies $w(B)+w\left(\tilde{T}_{i}\right) \geq 1$ and does not satisfy (1). Therefore we have

$$
w\left(\overline{\mathcal{B}_{i}}\right)+w\left(\tilde{T}_{i}\right)+w\left(v_{i}, \tilde{u}_{i}\right)>w(B)+w\left(\tilde{T}_{i}\right)+w\left(v_{i}, \tilde{u}_{i}\right)>\alpha .
$$

Then we obtain

$$
\begin{aligned}
4-\alpha=1+1+(2-\alpha) & >w\left(T_{i}\right)+w\left(\tilde{T}_{i}\right)+w\left(u_{i}, \tilde{u}_{i}\right) \\
& \geq\left(w\left(u_{i}, v_{i}\right)+w\left(B_{i}\right)+w\left(\overline{\mathcal{B}_{i}}\right)\right)+w\left(\tilde{T}_{i}\right)+w\left(u_{i}, \tilde{u}_{i}\right) \\
& \geq w\left(B_{i}\right)+w\left(\overline{\mathcal{B}_{i}}\right)+w\left(\tilde{T}_{i}\right)+w\left(v_{i}, \tilde{u}_{i}\right) \\
& >w\left(B_{i}\right)+\alpha,
\end{aligned}
$$

implying that $w\left(B_{i}\right)<4-2 \alpha$ holds. Therefore $B_{i} \in \mathcal{L}_{i}$ holds by the definition of $\mathcal{L}_{i}$. Then by the maximality of $B_{i}, w(B) \leq w\left(B_{i}\right)<4-2 \alpha$ holds for all $B \in \mathcal{B}\left(v_{i}\right)$. This implies that $\mathcal{H}_{i}=\emptyset$, which contradicts $\left|\mathcal{H}_{i} \cap \overline{\mathcal{B}_{i}}\right|>0$. Therefore $w\left(\overline{\mathcal{B}_{i}}\right)<2-\alpha$ holds.

Let $\left(T, z, T_{1}, T_{2}, u_{1}, u_{2}, p_{u_{1} v_{1}}, p_{u_{2} v_{2}}\right)$ denote a tuple for a 2-boundary vertex $z \in V_{2}(T)$ in a rooted tree $T$, two branches $T_{1} \in \mathcal{B}\left(u_{1}\right), T_{2} \in \mathcal{B}\left(u_{2}\right)$ rooted at $u_{1}, u_{2} \in V\left(T_{z}\right)$ (possibly $u_{1}=z, u_{2}=z$ or $u_{1}=u_{2}$ ), respectively, 1/2-boundary vertices $v_{1} \in V_{1 / 2}\left(T_{1}\right), v_{2} \in V_{1 / 2}\left(T_{2}\right)$, and (1/2)-distant 
points $p_{u_{1} v_{1}}, p_{u_{2} v_{2}}$ of $v_{1}, v_{2}$ with $u_{1}, u_{2}$, respectively. Assume that such a tuple $\left(T, z, T_{1}, T_{2}, u_{1}, u_{2}, p_{u_{1} v_{1}}, p_{u_{2} v_{2}}\right)$ satisfies Condition C. The following procedure, called Combine returns an 1-admissible set $S$, where a corresponding 1-admissible tree will be generated by introducing an edge which is not in the tree $T$.

Procedure Combine

Input: A tuple $\left(T, z, T_{1}, T_{2}, u_{1}, u_{2}, p_{u_{1} v_{1}}, p_{u_{2} v_{2}}\right)$ satisfying Condition C.

Output: An admissible set $S$ in $T$.

1 If $w\left(T_{v_{1}}\right)<1 / 2$ or $w\left(T_{v_{2}}\right)<1 / 2$ holds then

2 Return $S:=D\left(v_{1}\right) \cup D\left(v_{2}\right)$ (see Fig. 2 (a))

3 else $/ * w\left(T_{v_{1}}\right) \geq 1 / 2$ and $w\left(T_{v_{2}}\right) \geq 1 / 2$ hold $* /$

$4 \quad$ Choose a subset $\mathcal{S} \subset \mathcal{B}\left(v_{1}\right) \cup \mathcal{B}\left(v_{2}\right)$ such that

$1 \leq w(\mathcal{S})<3-\alpha$ holds (see Fig. 2 (b));

$5 \quad$ Return $S:=\mathcal{S}-\left\{v_{1}, v_{2}\right\}$

6 end. $/ *$ if $* /$

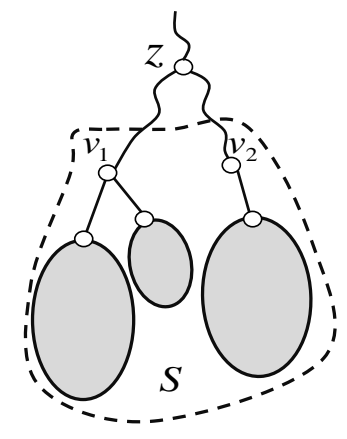

(a)

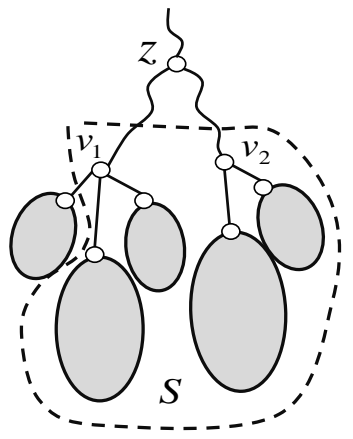

(b)

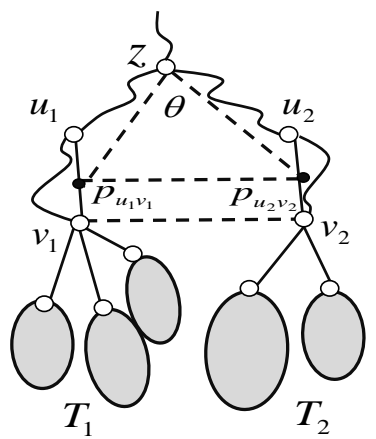

(c)

Figure 2: Introducing a shortcut $v_{1} v_{2}$. (a) $w\left(T_{v_{1}}\right)<1 / 2$ or $w\left(T_{v_{2}}\right)<1 / 2$; (b) $w\left(T_{v_{1}}\right) \geq 1 / 2$ and $w\left(T_{v_{2}}\right) \geq 1 / 2 ;$ (c) $\overline{v_{1} v_{2}}<\overline{p_{u_{1} v_{1} v_{1}}}+\overline{p_{u_{1} v_{1}} p_{u_{2} v_{2}}}+\overline{p_{u_{2} v_{2}} v_{2}}$,

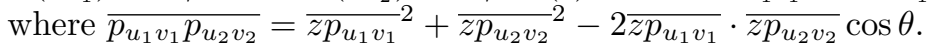

To show the correctness of Combine, we first discuss the following two lemmas.

Lemma 10 For a tuple $\left(T, z, T_{1}, T_{2}, u_{1}, u_{2}, p_{u_{1} v_{1}}, p_{u_{2} v_{2}}\right)$ satisfying Condition $C$, it holds

$$
\overline{v_{1} v_{2}}<2(5 / 2-\alpha) \sin (\theta / 2)+\overline{p_{u_{1} v_{1}} v_{1}}+\overline{p_{u_{2} v_{2} v_{2}}} .
$$


364 S. Karakawa, E. Morsy, and H. Nagamochi Minmax Tree Cover in the Euclidean Space

Proof: By the definition of (1/2)-distant points $p_{u_{1} v_{1}}$ and $p_{u_{2} v_{2}}$, Condition $\mathrm{C}(\mathrm{ii})$, and the cosine rule, we obtain (see Fig. 2(c))

$$
\begin{aligned}
{\overline{p_{u_{1} v_{1}} p_{u_{2} v_{2}}}}^{2} & ={\overline{z p_{u_{1} v_{1}}}}^{2}+{\overline{z p_{u_{2} v_{2}}}}^{2}-2 \overline{z p_{u_{1} v_{1}}} \cdot \overline{z p_{u_{2} v_{2}}} \cos \theta \\
& \leq\left(\overline{z u_{1}}+\overline{u_{1} p_{u_{1} v_{1}}}\right)^{2}+\left(\overline{z u_{2}}+\overline{u_{2} p_{u_{2} v_{2}}}\right)^{2}-2\left(\overline{z u_{1}}+\overline{u_{1} p_{u_{1} v_{1}}}\right)\left(\overline{z u_{2}}+\overline{u_{2} p_{u_{2} v_{2}}}\right) \cos \theta \\
& <(5 / 2-\alpha)^{2}+(5 / 2-\alpha)^{2}-2(5 / 2-\alpha)(5 / 2-\alpha) \cos \theta \\
& =4(5 / 2-\alpha)^{2} \sin ^{2}(\theta / 2) .
\end{aligned}
$$

This implies that $\overline{p_{u_{1} v_{1}} p_{u_{2} v_{2}}}<2(5 / 2-\alpha) \sin (\theta / 2)$. By the triangle inequality, we have

$$
\begin{aligned}
\overline{v_{1} v_{2}} & \leq \overline{p_{u_{1} v_{1}} p_{u_{2} v_{2}}}+\overline{p_{u_{1} v_{1} v_{1}}}+\overline{p_{u_{2} v_{2} v_{2}}} \\
& <2(5 / 2-\alpha) \sin (\theta / 2)+\overline{p_{u_{1} v_{1}} v_{1}}+\overline{p_{u_{2} v_{2}} v_{2}} .
\end{aligned}
$$

Lemma 11 For a tuple $\left(T, z, T_{1}, T_{2}, u_{1}, u_{2}, p_{u_{1} v_{1}}, p_{u_{2} v_{2}}\right)$ satisfying Condition $C$, the following (i), (ii) and (iii) hold:

(i) For each $i \in\{1,2\}$ satisfying $w\left(T_{v_{i}}\right)<1 / 2$, it holds $w\left(T_{v_{i}}\right)+\overline{p_{u_{i} v_{i} v_{i}}}<1 / 2$.

(ii) For each $i \in\{1,2\}$ satisfying $w\left(T_{v_{i}}\right) \geq 1 / 2$, it holds $w\left(T_{v_{i}}\right)<5 / 2-\alpha$ and $\overline{p_{u_{i} v_{i}} v_{i}}=0$.

(iii) If $w\left(T_{v_{1}}\right) \geq 1 / 2$ and $w\left(T_{v_{2}}\right) \geq 1 / 2$ hold, then there exists a subset $\mathcal{S} \subset$ $\mathcal{B}\left(v_{1}\right) \cup \mathcal{B}\left(v_{2}\right)$ such that $1 \leq w(\mathcal{S})<3-\alpha$ holds.

Proof: (i) If $\overline{u_{i} v_{i}}<1 / 2$ holds, then $\overline{p_{u_{i} v_{i} v_{i}}}=0$ holds by the definition of $p_{u_{i} v_{i}}$, implying that the statement is true. Otherwise, by Condition $\mathrm{C}(\mathrm{i})$, we have

$$
\begin{aligned}
1>w\left(T_{i}\right) & \geq w\left(T_{v_{i}}\right)+\overline{u_{i} v_{i}} \\
& =w\left(T_{v_{i}}\right)+\overline{u_{i} p_{u_{i} v_{i}}}+\overline{p_{u_{i} v_{i}} v_{i}} \\
& =w\left(T_{v_{i}}\right)+1 / 2+\overline{p_{u_{i} v_{i}} v_{i}} .
\end{aligned}
$$

Therefore we obtain $w\left(T_{v_{i}}\right)+\overline{p_{u_{i} v_{i}} v_{i}}<1 / 2$.

(ii) The former is trivial by Condition $\mathrm{C}(\mathrm{iv})$. To prove the latter, we show that $\overline{u_{i} v_{i}}<1 / 2$ holds. By Condition $\mathrm{C}(\mathrm{i})$, we have

$$
1>w\left(T_{i}\right) \geq w\left(T_{v_{i}}\right)+\overline{u_{i} v_{i}} \geq 1 / 2+\overline{u_{i} v_{i}},
$$

implying that $\overline{u_{i} v_{i}}<1 / 2$.

(iii) By Condition $\mathrm{C}(\mathrm{iv})$, we have a partition $\mathcal{B}\left(v_{1}\right) \cup \mathcal{B}\left(v_{2}\right)=\left\{B_{1}, B_{2}\right\} \cup$ $\overline{\mathcal{B}}_{1} \cup \overline{\mathcal{B}}_{2}$. We choose a minimal subset $\mathcal{S} \subset \mathcal{B}\left(v_{1}\right) \cup \mathcal{B}\left(v_{2}\right)$ such that $w(\mathcal{S}) \geq 1$ holds. Then by $w\left(\overline{\mathcal{B}}_{1}\right)<2-\alpha, w\left(\overline{\mathcal{B}}_{2}\right)<2-\alpha$ and $w\left(\mathcal{B}\left(v_{1}\right)\right)+w\left(\mathcal{B}\left(v_{2}\right)\right)=$ $w\left(T_{v_{1}}\right)+w\left(T_{v_{2}}\right) \geq 1$, the minimality of $\mathcal{S}$ implies $w(\mathcal{S})<1+(2-\alpha)=3-\alpha$.

Lemma 12 Any set $S$ output by ComBine is an admissible set. 
Proof: Obviously, $w(T)-w(T-S) \geq 1$ holds by $v_{1}, v_{2} \in V_{1 / 2}(T)$ and Lemma 11(iii). We also see that $T-S$ remains connected by construction of $S$. We claim that $T_{S}:=T\left[S \cup\left\{v_{1}, v_{2}\right\}\right]+\left(v_{1}, v_{2}\right)$ satisfies

$$
w\left(T_{S}\right)<3-\alpha+2(5 / 2-\alpha) \sin (\theta / 2)(\leq \alpha) .
$$

We distinguish the following three cases.

(i) $w\left(T_{v_{1}}\right)<1 / 2$ and $w\left(T_{v_{2}}\right)<1 / 2$. By Lemmas 10 and 11 we have

$$
\begin{aligned}
w\left(T_{S}\right) & =w\left(T_{v_{1}}\right)+w\left(T_{v_{2}}\right)+\overline{v_{1} v_{2}} \\
& <w\left(T_{v_{1}}\right)+w\left(T_{v_{2}}\right)+2(5 / 2-\alpha) \sin (\theta / 2)+\overline{p_{u_{1} v_{1}} v_{1}}+\overline{p_{u_{2} v_{2} v_{2}}} \\
& <1 / 2+1 / 2+2(5 / 2-\alpha) \sin (\theta / 2) \\
& <3-\alpha+2(5 / 2-\alpha) \sin (\theta / 2) .
\end{aligned}
$$

(ii) $\min \left\{w\left(T_{v_{1}}\right), w\left(T_{v_{2}}\right)\right\}<1 / 2 \leq \max \left\{w\left(T_{v_{1}}\right), w\left(T_{v_{2}}\right)\right\}$. We can assume that $w\left(T_{v_{1}}\right)<1 / 2$ and $w\left(T_{v_{2}}\right) \geq 1 / 2$ without loss of generality. Then by Lemmas 10 and 11 (i)-(ii), we obtain

$$
\begin{aligned}
w\left(T_{S}\right) & =w\left(T_{v_{1}}\right)+w\left(T_{v_{2}}\right)+\overline{v_{1} v_{2}} \\
& <w\left(T_{v_{1}}\right)+w\left(T_{v_{2}}\right)+2(5 / 2-\alpha) \sin (\theta / 2)+\overline{p_{u_{1} v_{1}} v_{1}}+\overline{p_{u_{2} v_{2} v_{2}}} \\
& <1 / 2+(5 / 2-\alpha)+2(5 / 2-\alpha) \sin (\theta / 2) \\
& =3-\alpha+2(5 / 2-\alpha) \sin (\theta / 2) .
\end{aligned}
$$

(iii) $w\left(T_{v_{1}}\right) \geq 1 / 2$ and $w\left(T_{v_{2}}\right) \geq 1 / 2$. Then by Lemmas 10, 11(ii)-(iii), we have

$$
\begin{aligned}
w\left(T_{S}\right) & =w(\mathcal{S})+\overline{v_{1} v_{2}} \\
& <w(\mathcal{S})+2(5 / 2-\alpha) \sin (\theta / 2)+\overline{p_{u_{1} v_{1} v_{1}}}+\overline{p_{u_{2} v_{2} v_{2}}} \\
& =3-\alpha+2(5 / 2-\alpha) \sin (\theta / 2) .
\end{aligned}
$$

Lemma 13 Let $T$ be a tree rooted at a vertex $r$ with $\operatorname{deg}(r)=1$ and has no active 1-boundary vertex. Let $\mathcal{S}$ be the admissible family in $T$ computed in Lemma 7 or Lemma 12. Then, for $T^{\prime}=T-\bigcup_{S \in \mathcal{S}} S$, if $T^{\prime} \neq \emptyset, w\left(T^{\prime}\right)=0$, and $w(T[S \cup\{r\}])>\alpha$ for all $S \in \mathcal{S}$, then $\mathcal{S} \cup V\left(T^{\prime}\right)$ is an admissible family in $T$.

Proof: Assume that $T^{\prime} \neq \emptyset, w\left(T^{\prime}\right)=0$, and $w(T[S \cup\{r\}])>\alpha$ for all $S \in \mathcal{S}$. Note that $T^{\prime} \neq \emptyset$ and $w\left(T^{\prime}\right)=0$ imply that $T^{\prime}=(\{r\}, \emptyset)$ in the Euclidean space. Clearly $w\left(T^{\prime}\right)=0<\alpha$. We observe that $T^{\prime}=(\{r\}, \emptyset)$ is obtained in Lemma 7(i)-(ii) where applied to a subtree $T_{z}$ for a 2-boundary vertex $z$ incident to $r$. Note that Lemma $7($ i) $/($ ii) computes a 2 -admissible family $\mathcal{S}=$ $\left\{S_{1}, S_{2}\right\}$ in $T$ such that $S_{1}=V\left(T_{1}\right), S_{2}=V\left(T_{z}\right)-S_{1}$, and $z \in S_{2}$ for a subtree $T_{1}$ with $w\left(T_{1}\right)>2 \alpha-3$. This implies that $T=T_{1}+T\left[S_{2} \cup\{r\}\right]$ holds. By assumption on the lemma, $w\left(T\left[S_{1} \cup\{r\}\right]\right)>\alpha$ holds. Therefore, $w(T)=w\left(T_{1}\right)+w\left(T\left[S_{2} \cup\{r\}\right]\right)>3 \alpha-3>2$ by assumptions on $\alpha=2 \sqrt{3}-3 / 2$. Thereby $\lceil w(T)\rceil \geq 3$, and hence $\mathcal{S} \cup\left\{V\left(T^{\prime}\right)\right\}$ is a 3 -admissible family in $T$. This proves the lemma.

Lemma 13 completes the proof of Theorem 6 
366 S. Karakawa, E. Morsy, and H. Nagamochi Minmax Tree Cover in the Euclidean Space

\section{Proof of Theorem 7}

The proof of Theorem 7 is similar to that of Theorem 6 described in the previous section. Let $T$ be a rooted tree that has no active 1-boundary vertices in $V_{1}(T)$. Then the main difference in the proof of Theorem 7 is the treatment of the case where $\left|\mathcal{H}^{*}\left(T_{z}\right)\right|=3$ and $\left|\mathcal{H}^{*}\left(T_{z}\right)\right| \geq 4$ for a 2-boundary vertex $z$ in $T$. We assume that $\alpha:=(13+\sqrt{109}) / 12$ throughout this section.

The next Lemma treats the case where $\left|\mathcal{H}^{*}\left(T_{z}\right)\right|=3$.

Lemma 14 For $z \in V_{2}(T)$ in a rooted tree $T$, let $\mathcal{H}^{*}\left(T_{z}\right)=\left\{T_{1}, T_{2}, T_{3}\right\}$. Let $u_{i} \in V_{1}(T) \cap V\left(T_{z}\right)$ and $v_{i} \in V_{1 / 2}\left(T_{i}\right)$ be the root and 1/2-boundary vertex of $T_{i}$, respectively, $i=1,2,3$. Then:

(a) For each $T_{i} \in\left\{T_{1}, T_{2}, T_{3}\right\}$, there exists a branch $\tilde{T}_{i} \in \mathcal{H}\left(\tilde{u}_{i}\right) \cap \mathcal{H}^{*}\left(T_{z}\right)$ such that the root $\tilde{u}_{i} \in V_{1}(T) \cap V\left(T_{z}\right)$ of $\tilde{T}_{i}$ satisfies $w\left(u_{i}, \tilde{u}_{i}\right)<2-\alpha$.

(b) For each $v_{i} \in\left\{v_{1}, v_{2}, v_{3}\right\}$, if $w\left(T_{v_{i}}\right) \geq 1 / 2$ holds and there exists a minimal subtree $\mathcal{S} \subseteq \mathcal{B}\left(v_{i}\right)$ satisfying $w(\mathcal{S})+w\left(\tilde{T}_{i}\right) \geq 1$ such that

$$
w(\mathcal{S})+w\left(\tilde{T}_{i}\right)+w\left(v_{i}, \tilde{u}_{i}\right) \leq \alpha
$$

holds, then $S:=\left(V\left(\tilde{T}_{i}\right)-\left\{\tilde{u}_{i}\right\}\right) \cup\left(V(\mathcal{S})-\left\{v_{i}\right\}\right)$ is an admissible. Testing whether such a subtree exists or not can be done in $O\left(\left|\mathcal{B}\left(v_{i}\right)\right|\right)$ time.

(c) If the condition of (b) does not hold, then there exists $\left\{T_{i}, T_{j}\right\} \subseteq\left\{T_{1}, T_{2}, T_{3}\right\}(i \neq$ $j)$ such that the tuple $\left(T, z, T_{i}, T_{j}, u_{i}, u_{j}, p_{u_{i} v_{i}}, p_{u_{j} v_{j}}\right)$ satisfies Condition $\mathrm{C}$ and

(v) $u_{i}=u_{j} \neq z$ or $z=u_{i}$ or $z=u_{j}$.

(vi) $\overline{v_{i} v_{j}}<\sqrt{1 / 4+(5 / 2-\alpha)^{2}-(5 / 2-\alpha) \cos \theta}+\overline{p_{u_{i} v_{i}} v_{i}}+\overline{p_{u_{j} v_{j}} v_{j}}$.

Proof: It suffices to show that (v) and (vi) hold.

(v) Since $\left|\mathcal{H}^{*}\left(T_{z}\right)\right|=3$ holds, $\left|V_{1}\left(T_{z}\right)\right| \leq 1$ by Condition $\mathrm{B}(\mathrm{ii})$. In this case, two of three branches in $\mathcal{H}^{*}\left(T_{z}\right)$ are rooted at some $u \in V_{1}(T) \cap V\left(T_{z}\right)$ (possibly $u=z$ ) and the other one branch must be rooted at $z$, as required.

(vi) We assume that $u_{i}=z$ and $u_{j} \neq z$ without loss of generality by (v). Similar to the proof of Lemma 10 , we have

$$
\begin{aligned}
{\overline{p_{u_{i} v_{i}} p_{u_{j} v_{j}}}}^{2} & ={\overline{z p_{u_{i} v_{i}}}}^{2}+{\overline{z p_{u_{j} v_{j}}}}^{2}-2 \cdot \overline{z p_{u_{i} v_{i}}} \cdot \overline{z p_{u_{j} v_{j}}} \cos \theta \\
& \leq{\overline{u_{i} p_{u_{i} v_{i}}}}^{2}+\left(\overline{z u_{j}}+\overline{u_{j} p_{u_{j} v_{j}}}\right)^{2}-2 \overline{u_{i} p_{u_{i} v_{i}}} \cdot\left(\overline{z u_{j}}+\overline{u_{j} p_{u_{j} v_{j}}}\right) \cos \theta \\
& <(1 / 2)^{2}+(5 / 2-\alpha)^{2}-2 \cdot 1 / 2 \cdot(5 / 2-\alpha) \cos \theta \\
& =1 / 4+(5 / 2-\alpha)^{2}-(5 / 2-\alpha) \cos \theta .
\end{aligned}
$$

This implies that $\overline{p_{u_{i} v_{i}} p_{u_{j} v_{j}}}<\sqrt{1 / 4+(5 / 2-\alpha)^{2}-(5 / 2-\alpha) \cos \theta}$. By the triangle inequality, we obtain

$$
\begin{aligned}
\overline{v_{i} v_{j}} & \leq \overline{p_{u_{i} v_{i}} p_{u_{j} v_{j}}}+\overline{p_{u_{i} v_{i}} v_{i}}+\overline{p_{u_{j} v_{j}} v_{j}} \\
& <\sqrt{1 / 4+(5 / 2-\alpha)^{2}-(5 / 2-\alpha) \cos \theta}+\overline{p_{u_{i} v_{i}} v_{i}}+\overline{p_{u_{j} v_{j}} v_{j}} .
\end{aligned}
$$


By Lemmas 11 and 14, as in the proof of Lemma 12, we can show that any output $S$ of ComBine is admissible because $T_{S}:=T\left[S \cup\left\{v_{i}, v_{j}\right\}\right]+\left(v_{i}, v_{j}\right)$ satisfies

$$
\begin{aligned}
w\left(T_{S}\right) & <(3-\alpha)+\sqrt{1 / 4+(5 / 2-\alpha)^{2}-(5 / 2-\alpha) \cos \theta} \\
& <(3-\alpha)+\sqrt{1 / 4+(5 / 2-\alpha)^{2}+(5 / 2-\alpha) \cdot 1 / 2} \\
& =\alpha .
\end{aligned}
$$

The next lemma treats the case where $\left|\mathcal{H}^{*}\left(T_{z}\right)\right| \geq 4$. In this case, obviously the minimum angle formed by four line segments $\overline{z v_{i}}(i=1,2,3,4)$ in $\mathbb{R}^{2}$ is no more than $90^{\circ}$.

Lemma 15 For $z \in V_{2}(T)$ in a rooted tree $T$, let $T_{1}, T_{2}, T_{3}, T_{4} \in \mathcal{H}^{*}\left(T_{z}\right)$. Let $u_{i} \in V\left(T_{z}\right)$ and $v_{i} \in V_{1 / 2}\left(T_{i}\right)$ be the root and 1/2-boundary vertex of $T_{i}$, respectively, $i=1,2,3,4$. Then:

(a) For each $T_{i} \in\left\{T_{1}, T_{2}, T_{3}, T_{4}\right\}$, there exists a branch $\tilde{T}_{i} \in \mathcal{H}\left(\tilde{u}_{i}\right) \cap \mathcal{H}^{*}\left(T_{z}\right)-$ $T_{i}$ such that the root $\tilde{u}_{i} \in V_{1}(T) \cap V\left(T_{z}\right)$ of $\tilde{T}_{i}$ satisfies $w\left(u_{i}, \tilde{u}_{i}\right)<2-\alpha$.

(b) For each $i \in\{1,2,3,4\}$, if $w\left(T_{v_{i}}\right) \geq 1 / 2$ holds and there exists a minimal subtree $\mathcal{S} \subseteq \mathcal{B}\left(v_{i}\right)$ satisfying $w(\mathcal{S})+w\left(\tilde{T}_{i}\right) \geq 1$ such that

$$
w(\mathcal{S})+w\left(\tilde{T}_{i}\right)+w\left(v_{i}, \tilde{u}_{i}\right) \leq \alpha
$$

holds, then $S:=\left(V\left(\tilde{T}_{i}\right)-\left\{\tilde{u}_{i}\right\}\right) \cup\left(V(\mathcal{S})-\left\{v_{i}\right\}\right)$ is admissible. Testing whether such a subtree exists or not can be done in $O\left(\left|\mathcal{B}\left(v_{i}\right)\right|\right)$ time.

(c) If the condition of (b) does not hold, then there exists $\left\{T_{i}, T_{j}\right\} \subseteq\left\{T_{1}, T_{2}, T_{3}, T_{4}\right\}$ ( $i \neq$ $j)$ such that the tuple $\left(T, z, T_{i}, T_{j}, u_{i}, u_{j}, p_{u_{i} v_{i}}, p_{u_{j} v_{j}}\right)$ satisfies Condition $\mathrm{C}$ with $\theta \leq 90^{\circ}$.

By Lemmas 11 and 15 and $\theta \leq 90^{\circ}$, as in the proof of Lemma 12 , we can show that the output $S$ of ComBine is admissible because $T_{S}:=T\left[S \cup\left\{v_{i}, v_{j}\right\}\right]+$ $\left(v_{i}, v_{j}\right)$ satisfies

$$
\begin{aligned}
w\left(T_{S}\right) & <(3-\alpha)+2(5 / 2-\alpha) \sin (\theta / 2) \\
& <(3-\alpha)+(5 / 2-\alpha) \cdot \sqrt{2} \\
& <\alpha .
\end{aligned}
$$

Note that the difference in the proofs of Theorem 6 and Theorem 7 is the treatment of the case where $\left|\mathcal{H}^{*}\left(T_{z}\right)\right|=3$ and $\left|\mathcal{H}^{*}\left(T_{z}\right)\right| \geq 4$ for a 2-boundary vertex $z$ in $T$. On the other hand, for $\left|\mathcal{H}^{*}\left(T_{z}\right)\right|=3$ and $\left|\mathcal{H}^{*}\left(T_{z}\right)\right| \geq 4$, a 1 -admissible family is computed such that the remaining tree has a nonzero weight. Therefore, the proof of the following lemma is the same as that of Lemma 13. 
368 S. Karakawa, E. Morsy, and H. Nagamochi Minmax Tree Cover in the Euclidean Space

Lemma 16 Let $T$ be a tree rooted at a vertex $r$ with $\operatorname{deg}(r)=1$ and has no active 1-boundary vertex. Let $\mathcal{S}$ be the admissible family in $T$ computed in this section. Then, for $T^{\prime}=T-\bigcup_{S \in \mathcal{S}} S$, if $T^{\prime} \neq \emptyset, w\left(T^{\prime}\right)=0$, and $w(T[S \cup\{r\}])>\alpha$ for all $S \in \mathcal{S}$, then $\mathcal{S} \cup V\left(T^{\prime}\right)$ is an admissible family in $T$.

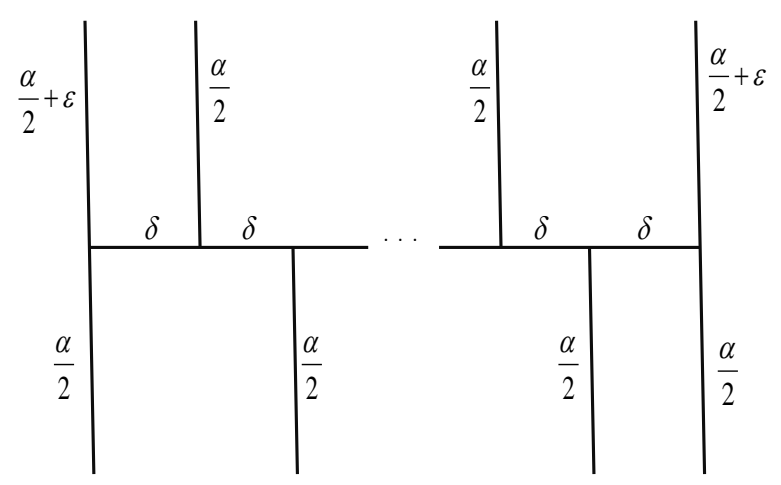

Figure 3: A minimum spanning tree $T^{*}$ of a given graph $G$ including $q$ horizontal line segments each of which has weight $\delta, q+1$ vertical line segments each of which has weight $\alpha / 2$, and each of the remaining two vertical line segments has weight $\alpha / 2+\varepsilon$, where $0<\alpha<2,0<\varepsilon<1-\alpha / 2, \delta=1-\alpha / 2-\varepsilon$, and $q=\max \{2,\lceil(3 \alpha-4) / 2 \varepsilon\rceil\}$. The weight of the tree is $q \delta+(q+1) \alpha / 2+(\alpha / 2+$ $\varepsilon) \times 2=q+\alpha+\alpha / 2-(q-2) \varepsilon \leq q+2$.

\section{Concluding Remarks}

In this paper, we have studied the balanced tree partitioning problem in the Euclidean space $\mathbb{R}^{d}$, and have shown that the ratio $\alpha$ of the maximum tree weight to $w\left(T^{*}\right) / k$ for the weight $w\left(T^{*}\right)$ of a minimum spanning tree is at most 1.965 for $d \geq 3$ and at most 1.954 for $d=2$, by designing polynomial time algorithms for finding a tree cover that attains such $\alpha$. To derive these results, we allowed for trees in a tree cover to use edges not in a minimum spanning tree $T^{*}$. We remark that if a tree cover is required to consist of subtrees of $T^{*}$, then the best possible $\alpha$ is at least 2. Such an example is shown in Fig. 3, where each line segment consists of numerous points in $\mathbb{R}^{2}$ and edges joining every two nearest points on it, and the whole set of edges in all line segments gives a (unique) minimum spanning tree $T^{*}$ for the set of all points in all line segments. For $q=\max \{2,\lceil(3 \alpha-4) / 2 \varepsilon\rceil\}$, it holds $w\left(T^{*}\right) \leq q+2$ since it contains $q+3$ vertical line segments each of which with weight of at least $\alpha / 2$. For $k=q+2$, 
any tree cover $\mathcal{T}$ which contains only subtrees of $T^{*}$ must contains a subtree $T \in \mathcal{T}$ with weight of at least $\alpha$. This implies that the ratio $w(T)$ to $w\left(T^{*}\right) / k$ is at least $\alpha$, which can be chosen as any number close to 2 .

It is left open to determine the best possible $\alpha$ for each dimension $d \geq 2$. For $d \geq 2$, we see that $\alpha$ cannot be smaller than 1.23 by the example in Fig. 4 (a) which consists of seven points $v_{1}, v_{2}, \ldots, v_{7}$ in $\mathbb{R}^{2}$ and a (unique) minimum spanning tree $T^{*}$ of $G$ is drawn by bold lines. It holds $w\left(T^{*}\right)<2$ (we see that $T^{*}$ is minimum because it can be constructed by choosing shortest edges repeatedly). For $k=2$, we show that an optimal tree cover $\mathcal{T}=\left\{T_{1}, T_{2}\right\}$ attains $\max \left\{w\left(T_{1}\right), w\left(T_{2}\right)\right\}=1.22$. For any tree cover $\left\{T_{1}, T_{2}\right\}$, two of the vertices $v_{1}, v_{2}$, and $v_{3}$ must be contained in one of $T_{1}$ and $T_{2}$; assume without loss of generality that $v_{1}, v_{2} \in V\left(T_{1}\right)$. Then either $V\left(T_{1}\right)=\left\{v_{1}, v_{2}\right\}$ or $V\left(T_{1}\right)=$ $\left\{v_{1}, v_{2}, v_{i}\right\}, i \in\{4,5\}$, since otherwise the weight of $T_{1}$ is larger than 1.22. Note that, in both cases, a minimum tree spanning vertices in $T_{2}$ is a subtree of $T^{*}$. Therefore, in the former case, $w\left(T_{1}\right)=1.14$ and $w\left(T_{2}\right)=1.22$, while in the latter case, $w\left(T_{2}\right)=0.94$ and a minimum tree spanning vertices in $T_{1}$ has weight 1.22. Thus, an optimal tree cover attains $\max \left\{w\left(T_{1}\right), w\left(T_{2}\right)\right\}=1.22$, and the ration of 1.22 to $w\left(T^{*}\right) / 2$ is at least 1.23 . It would be interesting and challenging to close the gap between the current upper and lower bounds on the ratio $\alpha$ by developing further insightful geometric arguments over the Euclidean space.

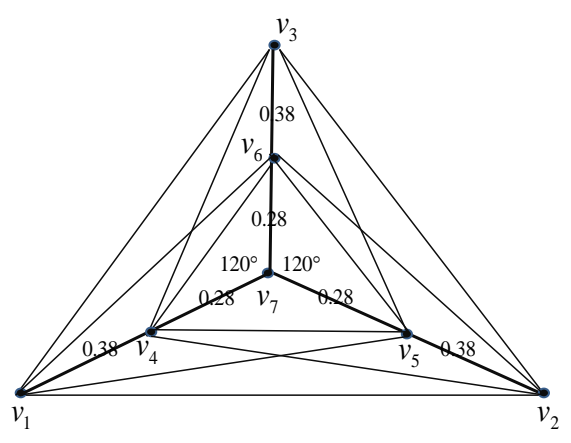

(a)

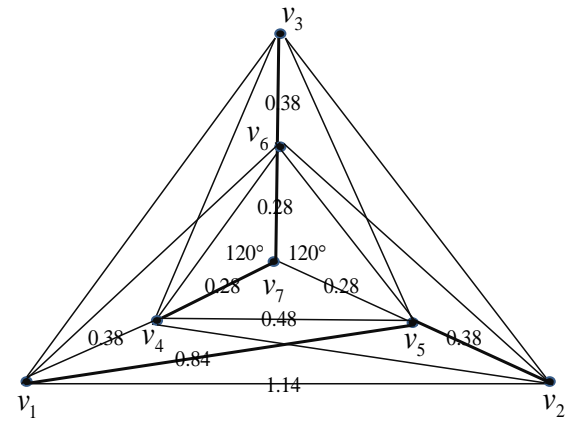

(b)

Figure 4: An example for showing a lower bound on the ration in the balanced tree partitioning problem. (a) Seven points in the Euclidean plane, where a minimum spanning tree $T^{*}$ of $G$ is drawn by bold lines; (b) An optimal tree cover for $k=2$ is drawn in bold lines. 
370 S. Karakawa, E. Morsy, and H. Nagamochi Minmax Tree Cover in the Euclidean Space

\section{References}

[1] I. Averbakh and O. Berman. A heuristic with worst-case analysis for minmax routing of two traveling salesman on a tree. Discrete Applied Mathematics, 68:17-32, 1996.

[2] I. Averbakh and O. Berman. $(p-1) /(p+1)$-approximate algorithm for $p$-traveling salesman problems on a tree with minmax objective. Discrete Applied Mathematics, 75:201-216, 1997.

[3] E. E. B. Bozkaya and G. Laporte. A tabu search heuristics and adaptive memory procedure for political districting. European J. Operation Research, 144:12-26, 2003.

[4] J. Bruno and P. Downey. Complexity of task sequencing with deadlines, set-up times and changeover costs. SIAM J. Comput., 7:393-404, 1978.

[5] R. Cordone and F. Maffioli. On the complexity of graph tree partition problems. Discrete Applied Mathematics, 134:51-65, 2004.

[6] J. K. R. R. G. Even, N. Garg and A. Sinha. Min-max tree covers of graphs. Operations Research Letters, 32:309-315, 2004.

[7] J. C. W. Jr. Political districting: a review. Papers in Regional Science, 74:12-40, 1995.

[8] Y. Karuno and H. Nagamochi. 2-approximation algorithms for the multivehicle scheduling on a path with release and handling times. Discrete Applied Mathematics, 129:433-447, 2003.

[9] Y. Karuno and H. Nagamochi. Scheduling vehicles on trees. Pacific J. Optim., 1:527-543, 2005.

[10] Y. Karuno and H. Nagamochi. Vehicle scheduling problems in graphs. Handbook of Approximation Algorithms and Metaheuristics, Chapman $\mathcal{E}$ Hall/CRC, Boca Raton, Chapter 46, 2007.

[11] C. L. M. Andersson, J. Gudmundsson and G. Narasimhan. Balanced partition of minimum spanning trees. International Journal of Computational Geometry and Applications, 13:303-316, 2003.

[12] H. Nagamochi. Approximating the minmax rooted-subtree cover problem. IEICE Trans. Fundamentals, E88-A, No.5:1335-1338, 2005.

[13] H. Nagamochi and T. Kawada. Minmax subtree cover problem on cacti. Discrete Applied Mathematics, 154(8):1254-1263, 2006.

[14] H. Nagamochi and K. Okada. Polynomial time 2-approximation algorithms for the minmax subtree cover problem. In Proceedings of The 14th Annual International Symposium on Algorithms and Computation (ISAAC2003), LNCS 2906:138-147, 2003. 
[15] H. Nagamochi and K. Okada. A faster 2-approximation algorithm for the minmax $p$-traveling salesman problem on a tree. Discrete Applied Mathematics, 140:103-114, 2004.

[16] H. Nagamochi and K. Okada. Approximating the minmax rooted-tree cover in a tree. Information Processing Letters, 104:173-178, 2007. 